\title{
Determination of the Tolerable Impurity Concentrations in a Fusion Reactor using a Consistent Set of Cooling Factors
}

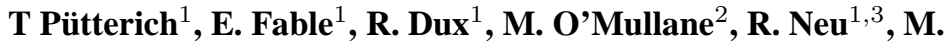 \\ Siccinio $^{4}$ \\ ${ }^{1}$ Max-Planck-Institut für Plasmaphysik, EURATOM Association, D-85748 Garching, \\ Germany \\ ${ }^{2}$ CCFE, Culham Science Centre, Abingdon, Oxfordshire OX14 3DB, United Kingdom \\ ${ }^{3}$ Technische Universität München, 85748 Garching, Germany \\ ${ }^{4}$ EUROfusion Programme Management Unit, 85748 Garching, Germany
}

\begin{abstract}
In the present work, the tolerable impurity level and composition for a reactor plasma using several sets of model assumptions are evaluated. Special care was taken to evaluate a comprehensive and consistent set of atomic data for 35 different elements, such that the impurity level for various elements may be studied as a function of their nuclear charge. The data set may not only be useful for the presented work or for system codes which design fusion reactors, but also for interpretation of bolometric measurements. Additionally, the predictions of the spectral distribution of the radiated power is of high quality such that soft X-ray broadband measurements may be interpreted.

In the present work the data is used for predicting the radiated power in a reactor plasma, using a 0D, several variants of a $0.5 \mathrm{D}$ model and a realistic 1D ASTRA modelling of a DEMO plasma, i.e. the EU DEMO1 2015 design. The maximal or appropriate impurity content of a reactor plasma for all models can be determined, such that the predictions from a simplistic OD model can be compared to less simplistic models and a proper reactor simulation. These comparisons suggest that with the simplistic models the impurity content may be estimated within a factor of about 1.5 , independent of the realization of the reactor plasma. At the same time this study underlines the sensitivity of the reactor performance on the impurity mixture and especially of the He content of the plasma. Additionally, an extended 0.5D model is presented which is able to predict variations of the fusion yield $Q$ and the He concentration, when both is known for a reference scenario. These predictions prove to be of high accuracy when compared to the 1D ASTRA modelling and thus, allow the net impact of an increased dilution and a simlutaneous temperature rise at constant plasma pressure to be evaluated. Furthermore, the parameter space is scanned with more than $10^{5}$ model reactor plasmas demonstrating that the use of a low- $\mathrm{Z}$ impurity diminishes the possibility of an economical feasible reactor plasma. The main results of the parameter scan are made available via scaling formulae.
\end{abstract}

PACS numbers: $28.52,32.30,34.80,52.20,52.25,52.40,52.55$

Nuclear Fusion

E-mail: Thomas.Puetterich@ipp.mpg.de

\section{Introduction}

In todays fusion experiments impurities are often considered a nuisance, levels of $Z_{\text {eff }}$ are minimized and the radiated power is considered missing power when stability and performance of a plasma discharge are evaluated. At the same time it is well known that in a reactor impurities are needed in order to radiate the power rather than guiding it into the 
divertor. Both views are justified and for a reactor the right balance between beneficial and detrimental effects is required, thus, an optimal impurity level and composition exists. This problem is multifaceted and in the present work the focus is put on the balance of fusion performance and radiative cooling inside the last closed flux surface.

A crucial ingredient for investigations of this topic are the atomic data necessary for evaluating the radiative cooling of an impurity. While being crucial, a study of the literature reveals that the necessary atomic data is either of baseline quality [1] or it is available for only a few elements in high quality [2]. Additionally, the quality standards for low- $Z$ elements are typically much higher than for high- $Z$ elements, as the latter are characterized by more complex electronic structures making the structure calculation and all subsequent calculations such as those of collision cross-sections and collisional-radiative modelling more complicated and thereby limited. Moreover, an evaluation of radiative cooling requires atomic data for all ion stages of an element, which makes heavy elements due to their many ion stages doubly challenging. Due to these limitations the most up to date atomic data of e.g. tungsten (W) from [3-8] are less advanced than the calculation available for low-Z elements (e.g. [2]). Still, the modern calculations deal with the details of the wave functions such that the prediction of realistic spectra is possible $[3,4,6]$ also enabling the interpretation of broadband measurements of soft X-ray radiation. Generally the best atomic data should be used for all applications, which implies that for various elements a mix of sources for the atomic data is necessary. However, this implies that certain effects in the studied physics, e.g. impurity limits in a fusion reactor, may originate not only from the characteristics of studied elements, but also from the characteristics of the used calculation method. The data from different sources may be evaluated via different optimization criteria and plasma parameter grids such that an application of the data in a different context is either not appropriate or requires extrapolation of the temperature or density grids. Such an effect seems visible in figure 1 of [9], where clearly not all cooling curves follow a $\sqrt{T_{e}}$-dependence for high temperatures, indicating that in this temperuture range the Bremsstrahlung contribution is not correctly evaluated for all cases. This effect is also discussed in [9]. In order to provide a data set, which features as high quality as possible and at the same time is of similar quality for all considered elements a new data set has been evaluated using the same codes and the same complexity for all ion stages of all elements. These data sets are depicted in figure 1 and are also made available following the publication of the present work.

The atomic data are then applied in several types of reactor models in order to determine the optimal impurity level for all of the considered impurities. The studied reactor models are several simple models in which a balance between heating of the plasma via the $\alpha$-particles produced by DT-fusion and all losses is assumed. Stepwisely the complexity of the model is increased leading to a better traceability of model assumptions and their effect. The last enhancement gives insights into the effect of the impurities on the fusion power, He content and radiative cooling, while all parameters fullfill self-consistently the above mentioned power balance. These values are then compared to a transport model, i.e. a 1D ASTRA simulation based on the EU DEMO1 2015 design [10]. Furthermore, the most enhanced of the power balance models allows to predict quickly the impurity content and other important parameters, which are of interest when considering a fusion reactor of any kind. Thus, it is used to provide a huge database of reactor plasmas properties which are then made available in terms of scaling formulae. 


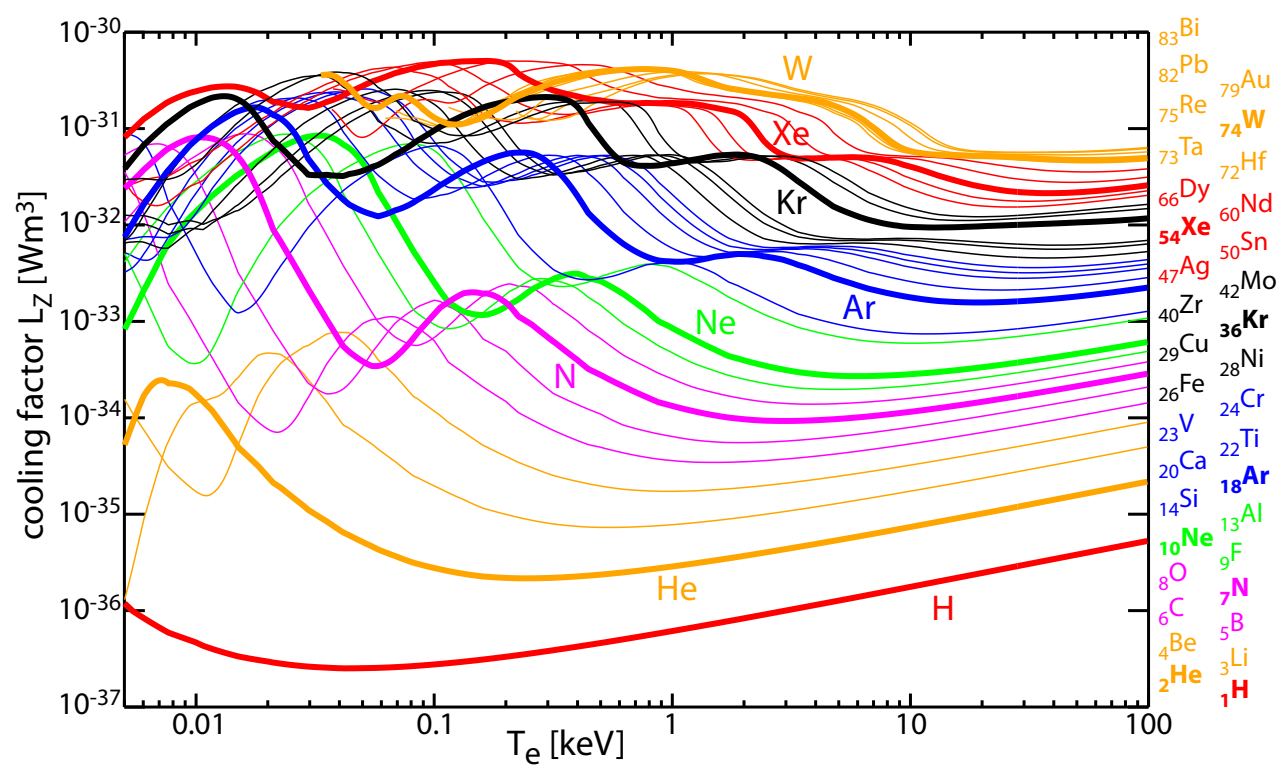

Figure 1. (colored in online-version) Calculated cooling factors for the considered elements from $\mathrm{H}$ to $\mathrm{Bi}$ at $n_{e}=5 \cdot 10^{19} \mathrm{~m}^{-3}$. All atomic data will be made available via ADAS.

\section{Radiation Losses and Atomic Data}

As the optimal impurity level crucially depends on the radiative cooling by an impurity, the related atomic data is of major importance for the following investigations. This article focusses on the implications on the impurity level in a reactor device and thus, the description and focus of the atomic data is limited and presented elsewhere. Nevertheless a basic description of the considered atomic data and related radiation losses is presented along a brief description of synchrotron radiation losses. It should be noted that the atomic data produced feature the following characteristics:

- The focus is put on the temperature range of the confined plasma, i.e. $\sim 100-200 \mathrm{eV}<$ $\mathrm{T}_{\mathrm{e}}<100 \mathrm{keV}$, which is a temperature range excluding neutrals and lowly charged ion stages notorious for computational and model complications.

- The calculated data for highly radiating species in this $T_{e}$-range, i.e. mid-Z to high-Z elements, is of elevated but not cutting edge quality. In last consequence it means that its quality has been assessed before, and the calculation procedure is not new. The advantage of the new data is rather the fact that for each ion the same codes and approximations are used.

- The line radiation is obtained via calculations with the Cowan code using the plane-wave Born approximation and an extrapolations to large quantum numbers.

- The Bremsstrahlung is obtained via the original formulae from [11] and the interpolation of Gaunt factors is done using the parametrization from [12]. For low-Z elements this contribution is dominant.

- The ionization rates are obtained via the configuration averaged distorted wave scheme in ADAS [13] similar to that presented in [14]. 
- The recombination rates are obtained from a parametric form implemented in adas $407 / 408$ in ADAS [13]

- The new atomic data is called 'consistent' across different impurity elements, because not only line radiation but also Bremsstrahlung and recombination radiation is evaluated using the same formulae and Gaunt factors. The used temperature grid was chosen such that no extrapolations are performed. Additionally also the ionization equilibria do behave systematic from element to element. The calculations are performed for more than 1000 different ions.

\subsection{Line Radiation}

For ion stages with bound electrons typically line radiation is the dominant fraction of the total radiated power. As the calculation of line radiation is also connected to many choices, a detailed description of all undertaken steps is presented in the following.

2.1.1. Electronic Structure The electronic structure of ions is given by the properties of the wave functions of all levels. In this context, a 'level' corresponds to the finest discrimination between wave functions taking the differences in angular momentum and radial distribution of the wave functions amplitudes into account. For the calculation of the unknown wave functions a scheme is used called multi-configuration Hartree-Fock (MCHF), which models the unknown wave function by a linear combination of basis functions obtained by the multiplication of hydrogenic orbitals. These (because they are hydrogenic) wave functions are well-known. More details can be found in [15]. Note, that the calculated structure exhibits as many levels as the number of levels in the basis and the accuracy and the properties of the structure depend strongly on the basis functions used. For example, if the structure for $\mathrm{Cu}$-like $\mathrm{W}^{45+}$ is calculated by using all levels contained in the configuration $1 s^{2} 2 s^{2} 2 p^{6} 3 s^{2} 3 p^{6} 3 d^{10} 4 s^{1}$ as a basis (which are actually just two levels, given by the different spin of the electron) only two unknown wave functions will be described. This will not yield a good description of the spectrum, e.g. dipole transitions require a parity change of the involved wave functions. Clearly, a rather large basis is necessary to describe the structure of any $\mathrm{W}$ ion well. Note that for a complete description the basis must be infinitely large implying that always a choice has to be made when performing calculations on atomic structure. The choice is typically aiming on describing the levels of interest well. For the presented calculations a choice performed for the calculation of the $\mathrm{W}$ spectra presented in [5] is underlying. The same choice is kept for all isoelectronic ions. In [4] and [5], the choice of levels was optimized to describe the most intense lines and to maximize the radiative power from each ion, while staying within the limits of the Cowan code [15].

After the wave functions are available, the transition probabilities for all sorts of spontaneous transitions can be calculated, typically involving a choice about the type of considered transitions. In the presented calculations electric and magnetic dipole and quadrupole transitions have been considered.

2.1.2. Plane-wave Born Approximation for Electron Impact Excitation In order to predict the radiation of the impurity ions, the calculation of electron collision cross sections is necessary. This calculation is computationally challenging, because any two levels of an impurity ion may be linked via an electron collision, while the cross-section calculation needs to be performed at many energies in order to calculate rates in a thermal plasma. A prerequisite for the cross-section calculation is the knowledge of the ion structure (cf. above 
paragraph), i.e. the wave function of each level. These wave functions are then combined with that of the colliding electron in order to quantify the excitation probabilities and thus, cross-sections. For the excitation process there are different approximations, one of which is the plane-wave Born-approximation. In this scheme the incoming and outgoing electron are treated as plane waves. The difference in the wave vectors corresponds to a plane wave that is combined with the wave functions of each bound level in order to find the characteristics of the wave functions corresponding to excited bound levels. The plane-wave Born approximation works well at high collision energies, where the wave function of the colliding electron is not strongly perturbed by the target ion. A shortcoming of the plane-wave Born approximation is that the colliding electron is always distinguishable from the bound electrons and thus spin changing transitions are not possible within this scheme. Still, for high-Z elements this shortcoming is less of a limitation as the quantum number 'Spin' of a single electron is not a well-defined quantum number for highly charged ions. This calculation scheme is implemented via adas801, i.e. the Cowan code within ADAS [13]. Note, that for high-Z elements strongly forbidden transitions beyond quadrupole may become relevant and cannot be treated via the the Cowan code, which is expected to have limited impact on the cooling factor.

2.1.3. Collisional Radiative Model For typical electron densities in the confined part of fusion plasmas the zero density approximation, assuming that all excitation is performed from the ground state (Coronal Approximation), is generally not sufficient, as excited populations - especially in metastable states - may yield additional excitation channels. Additionally, the metastable systems may hold a considerable part of the total population of an ion such that its existence influences the total radiated power from the ion. Thus, the application of a so-called collisional-radiative model is required, which considers the rate equations for each populated state. The rate equation for an excited level $\mathrm{k}$ contains sinks due to collisions, i.e. de-excitation and excitation away from the considered level $\mathrm{k}$, and due to spontaneous emission, described by the associated Einstein-coeffcients. The sources are given by collisional de-excitation and excitation into the level $\mathrm{k}$ and by cascades from higher levels, i.e. spontaneous emissions connecting to the level $\mathrm{k}$. This system of rate equations is solved via adas810 in ADAS. It may be noted that the mentioned processes responsible for the population sinks and sources of a level $\mathrm{k}$, are the absolute minimum for the collisional radiative modelling. Further processes like excitation due to inner-shell ionization or populations following radiative recombination may be added if necessary. However, the time scales of ionization and recombination are typically a few orders of magnitude slower than the time scales for excitation and spontaneous emission such that the rate equations for the populations of levels within each ion stage may be separated from the ionization equilibrium.

2.1.4. Choice of Level-Resolved or Configuration Averaged A level-resolved calculation has the advantage that the calculated line power can be easily compared with spectral meausurements and a detailed benchmark on the spectral distribution of the radiated power may be performed. In [4] such a benchmark for level-resolved W-data has been performed and even though the typical calibration uncertainties of the experimental data are in the range of 50\%, the fact that the most intense emissions of the $\mathrm{W}$ ions are found in the modelled spectra is quite encouraging. However, a comparison between level-resolved (LR) data to data from configuration averaged (CA) calculations (cf. [5]) revealed, that the total radiated power as calculated from LR data is well matched by the CA calculation, if the same input configurations for both calculations are used. At the same time the computational size and 
demand is much smaller for the CA calculations allowing for an increased number of included configurations. For W ions with partially filled $4 \mathrm{f}$ - or $4 \mathrm{~d}-$ or $3 \mathrm{~d}$-shells, this larger number of configurations made a considerable difference (cf. [5], about a factor of 2 for the radiated power) as the number of configurations for the LR calculations was chosen rather small, in order to keep the system computationally manageable. These observations suggest that when calculating the cooling factor for high- $Z$ elements it is not advisable to make compromises with the number of included configurations, but rather one should move to a CA calculation. Additionally, it is found that for Ar-like, Kr-like or Pd-like ions the lowest excited levels have a large difference in $\mathrm{J}$ to the ground state for LR calculations, which hinders the radiative decay unless alternative decay channels are included in the calculation. This effect is not an issue for CA and thus, if not a large enough model is implemented for an LR calculation the CA calculation is a better compromise. For the present work, a LR and CA data set was calculated, each including sets of configurations which have been identified in [4] (LR) and [5] (CA) to be of highest importance for the spectra (LR) and total radiated power (CA), respectively. Note, that this choice was performed via 'trial and error' and not via an algorithm (e.g. [16]).

2.1.5. Top-up Corrections The LR data may be considered irrelevant for the present work, but it has large potential for spectral modelling and also for benchmarking the CA data. Also, if the data is used for the interpretation of broadband soft X-ray emissions the LR data is potentially superior. Ideally, the LR data is used and the missing transitions are taken from the CA calculation and then added on top of the LR transitions. Such a procedure assumes that the structure, the excitation and also the collisional radiative modelling behaves linearly for additional transitions, which is not guaranteed and in fact, often not expected. Nevertheless, this procedure may be surveilled by benchmarking the result with an equivalent, pure CA calculation. For the presented data sets such a comparison was performed for serveral key impurities and the approach of topping up the LR data with additional transitions from the CA calculation ended up in cooling factors that were less than $20 \%$ different from each other.

Both calculations, i.e. LR and CA, lack the contributions of high-n transitions. Considering electron impact excitation as the main driver of the excitation processes, the importance of high-n transitions should be less pronounced the higher the principal quantum number $\mathrm{n}$ of the excited state is. In the present work it is attempted to estimate the missing power from such transitions by simply employing a $n^{-3}$ scaling of the exciation cross sections. Note, that this approximation is valid for hydrogenic species and breaks down when approaching the valence shell. Nevertheless it is used here as a mean to estimate the missing radiation. This top-up correction is smaller than $10 \%$ for the relevant temperature ranges and becomes larger (up to $20 \%$ ) only for lowly charged ions of high-Z elements which exist below $100 \mathrm{eV}$. Thus, this correction is not important for the present work, while it is still taken into account.

2.1.6. Ingredients of the Final Consistent Data Set Used for the Evaluation of the Cooling Factors The final data sets feature the same configurations for all isoelectronic ions of all impurities. For all cooling factors the CA calculations using a maximized set of configurations (cf. to [5]) was used. As the configuration sets are the same across an isoelectronic sequence also the high-n corrections are the same. Note, that also the code implementations for the evaluation of the other contributions (cf. sections below) to the cooling factor are kept the same for all ions. Therefore, we call the data 'consistent'. However, this procedure does not allow for high-quality evaluation of the data for low-Z elements. This is tolerable, because for 
low-Z elements the major effect on the reactor core plasma arises from Bremsstrahlung and dilution, which both do not benefit from high-quality methods applicable to ions which are not fully-stripped. For the temperatures relevant for the confined reactor plasmas, an even more systematic choice of configurations could be beneficial (e.g. [16]). However, when comparing the high temperature data for the elements presented in (e.g. [16]) to the data presented in the present work the differences are smaller than $\approx 30 \%$.

Note, as indicated several times that LR data was calculated along the CA data and it makes sense to also provide atomic data for the interpretation of measurements from soft Xray cameras. These data will be made available via ADAS or via direct communication, but are not further discussed in the present work.

\subsection{Ionization Equilibrium}

The ionization rates are calculated using the code adas802 within the ADAS [13] framework. Adas802 uses a configuration averaged distorted wave (CADW) scheme which is almost identical to the one presented in [14]. The resulting ionization cross sections are considered to be of high-quality, however, for the purpose of large-scale data production, the calculation of ionization rates ignores effects due to finite electron density, i.e. only ionization from the ground state is taken into account. The recombination rates are calculated using the case A implementation in adas407/408. The case A implementation uses a hydrogenic model for the radiative recombination. For the dielectronic recombination, it uses Burgess' general formula [17]. This approach obtains the recombination rates via parametric forms, into which level energies enter. Within ADAS an improved case B implementation is available, which is a slight improvement for the radiative recombination and which uses the Burgess Bethe General Program [18] for the dielectronic recombination, however, in the present work case A was used as a nummerically more stable choice. A systematic comparison between case A and case B is presented in [6]. Note that for the application of adas407/408, the electronic structure of all ions needs to be available, the calculation of which is described above.

\subsection{Radiation due to Recombination and Bremsstrahlung}

For the major part of the electron temperature range under consideration the radiation from recombination is only a small fraction of the total radiation, while at high electron temperatures the Bremsstrahlung becomes important if not dominant. The contribution of Bremsstrahlung is calculated using the formula of [1] and the free-free Gaunt factor of [11]. The later is used from the parametrisation presented in [12], because it best covers the desired parameter space also for spectral distributions. From the ADAS codes used to evaluate radiative and dielectronic recombination, also radiative powers related to these processes are evaluated. The spectral distribution of radiative recombination is given by the Maxwellian distribution of the recombining electrons, while for the dielectronic case the assumption is employed that each recombination emitts 1.2 times the ionization potential of the recombining ion. Both, the radiation due to recombination and the Bremsstrahlung are spectrally resolved such that the spectral interpretation is possible. For the dielectronic recombination it is assumed that the photons have an energy of approximately 0.75 the ionization potential of the recombining ion. This is a rought estimate and should be considered good enough only for cases in which the photon emission is not dominated by dielectronic recombination. For all considered elements, the ionization equilibria are such that this assumption holds. 


\subsection{Synchrotron Radiation}

In a magnetically confined fusion reactor, hot electrons are forced on curved trajectories via magnetic fields. Under these conditions electrons are known to emit synchrotron radiation and some part of that is reabsorbed and subject of radiation transport within the plasma volume. As a result the exact synchrotron radiation losses can only be evaluated, if knowledge about the magnetic field strength, the plasma temperature and density and the plasma geometry are known [19]. Approximations to these formulae are possible (e.g. [20]), but at least require the knowledge of the magnetic field, plasma temperature and density. Considerations including the parameter dependencies of the synchrotron radiation allow for restriction in the plane of magnetic field versus large radius of the device [20]. However, the metric used in the present work focusses on the plane of fusion tripple product versus temperature and thus, the parameter dependencies of the synchrotron losses can not be included in the results. At one point of this plane several values of the synchrotron radiation could apply depending on the details of the reactor design. The presented considerations on a reactor are still valid if including the synchrotron losses explicitly. The latter is crucial if synchrotron losses are a dominant loss mechanism. The synchrotron losses can be calculated via the formulae presented in [19].

\section{Results For The Investigated Reactor Models}

In the following all models are described, their results are presented and then discussed in the context of the earlier results. At the end of this section, the observations of all models are summarized and discussed together, as this yields insight into the credibility of the simple models applicable to reactor plasmas of arbitrary size.

\subsection{OD Power Density Balance with flexibility in the $n T \tau_{E} v s . T$ plane}

The investigated 0D model corresponds to case 4 in section 3 of [21]. For simplicity, we also introduce the total hydrogen concentration

$$
c_{D+T}=\frac{n_{\text {Deuterium }}+n_{\text {Tritium }}}{n_{e}}=1-q c_{Z}-2 c_{H e}
$$

and the ratio of total particle density over the electron density $n_{e}$, i.e.

$$
c_{\text {tot }}=\frac{n_{e}+n_{\text {Deuterium }}+n_{\text {Tritium }}+n_{H e}+n_{Z}}{n_{e}}=2-(q-1) c_{Z}-c_{H e}
$$

where $q$ is the charge of the impurity with nuclear charge $Z, n_{\text {Deuterium }}$ is the deuterium (D) density, $n_{\text {Tritium }}$ is the tritium (T) density, $n_{H e}$ is the helium (He) density, $n_{Z}$ is the density of the impurity with nuclear charge $Z$ and $c_{H e}$ and $c_{Z}$ are the respective impurity concentrations, i.e. the ratio of their ion density over $n_{e}$. For obvious reasons it is assumed that $n_{\text {Deuterium }}=n_{\text {Tritium }}$ in the reactor plasma.

The model balances the heating density by $\alpha$-particles $\left(P_{f u s}\right)$ with the loss power densities due to radiation $\left(P_{\text {rad }}\right)$ and transport $\left(P_{\text {transp }}\right)$. Including He and an other impurity $Z$ their effect on dilution and radiation the balance

$$
P_{f u s}=P_{r a d}+P_{t r a n s p}
$$


becomes:

$$
\begin{array}{ll}
\left(\frac{c_{D+T} n_{e}}{2}\right)^{2}\langle\sigma v\rangle E_{\alpha}= & \text { (fusion heating) } \\
=c_{D+T} n_{e}^{2} L_{H}+c_{Z} n_{e}^{2} L_{Z}+c_{H e} n_{e}^{2} L_{H e} & \text { (radiation losses) } \\
+\frac{\frac{3}{2} k_{B} T n_{e} c_{t o t}}{\tau_{E}} & \text { (transport losses) }
\end{array}
$$

where $\langle\sigma v\rangle$ is the Maxwell-averaged DT reaction parameter (used from [22]), $E_{\alpha}$ is the $\alpha$ energy, i.e. $3.52 \mathrm{MeV}, L_{Z}$ is the cooling rate coefficient of the element $Z, k_{B}$ is Boltzmann's constant and $\tau_{E}$ is the energy confinement time describing the energy loss via transport only. This balance provides the so-called burn condition and in the plane $n T \tau_{E}$ vs. $T$ it is matched on the so-called burn curve.

In order to treat the He ash self-consistently, it is assumed that the He confinement time $\tau_{H e}^{*}$, which is including recycling effects, is proportional to $\tau_{E}$ (cf. [21]). The following balance of the He source and sink determines the He level present in the reactor plasma.

$$
\left(\frac{c_{D+T} n_{e}}{2}\right)^{2}\langle\sigma v\rangle=\frac{c_{H e} n_{e}}{\tau_{H e}^{*}} \quad \text { (He balance) }
$$

For a given ratio $\rho^{*}=\tau_{H e}^{*} / \tau_{E}$ and for a given level of an additional impurity $Z$, the He concentration is first evaluated from a cubic equation as presented in [21] and then the power balance between sources and losses is evaluated for many grid points in the $n T \tau_{E}$ versus $T$ plane. At the points where the losses are equal to the sources, the burn condition is fullfilled and the burn curve is found. Note that for the $0 \mathrm{D}$ approach the ratio $\rho^{*}=\tau_{H e}^{*} / \tau_{E}$ must stay below 15.2 in order for a burn condition to exist, even if no other impurities are present. In experiment values of $\rho^{*}$ down to $\approx 4$ have been observed [23].

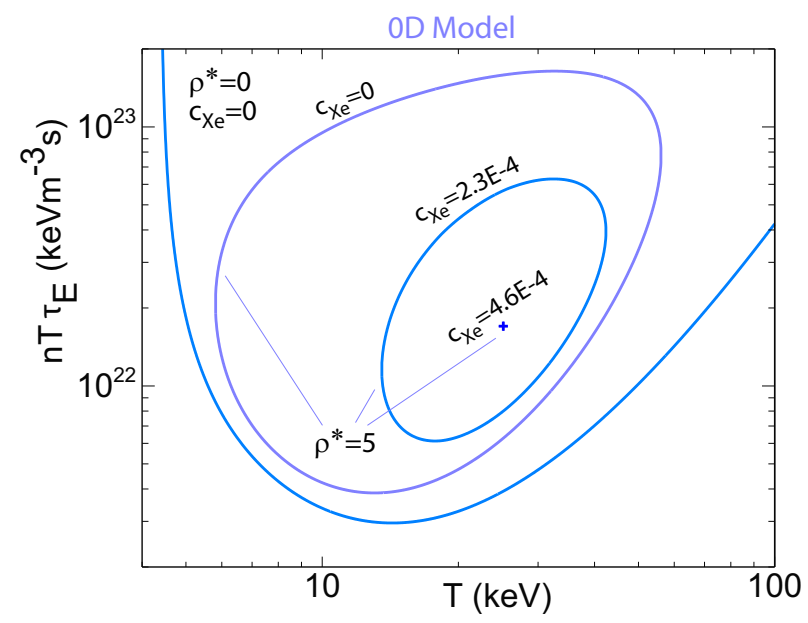

Figure 2. Burn curves for Xe resulting from a OD power balance for various the confinement time ratios $\rho^{*}=\tau_{H e}^{*} / \tau_{E}$ and Xe concentrations.

For obtaining figure 2, the above described process was performed for $\rho^{*}=0$ and $\rho^{*}=5$ and xenon $(\mathrm{Xe})$ at a concentration of $0,2.3 \cdot 10^{-4}$ and $4.6 \cdot 10^{-4}$. For $\rho^{*}=5$ the operational space included by the burn curve is smaller than for $\rho^{*}=0$ demonstrating the detrimental effect of the He ash. Similarly, the operational space becomes smaller for an 
increasing amount of $\mathrm{Xe}$, such that for a Xe concentration above $4.6 \cdot 10^{-4}$ the burn condition cannot be fullfilled at $\rho^{*}=5$. Thus, $4.6 \cdot 10^{-4}$ is the maximum Xe concentration for $\rho^{*}=5$.

In the present work the maximum impurity concentration is determined for all impurities for which cooling factors have been calculated at various $\rho^{*}$. In figure 3(a), all maximum impurity levels are given for a range of $\rho^{*}$ from 0.5 to 14 . For small $\rho^{*}$ the ratio of tolerable

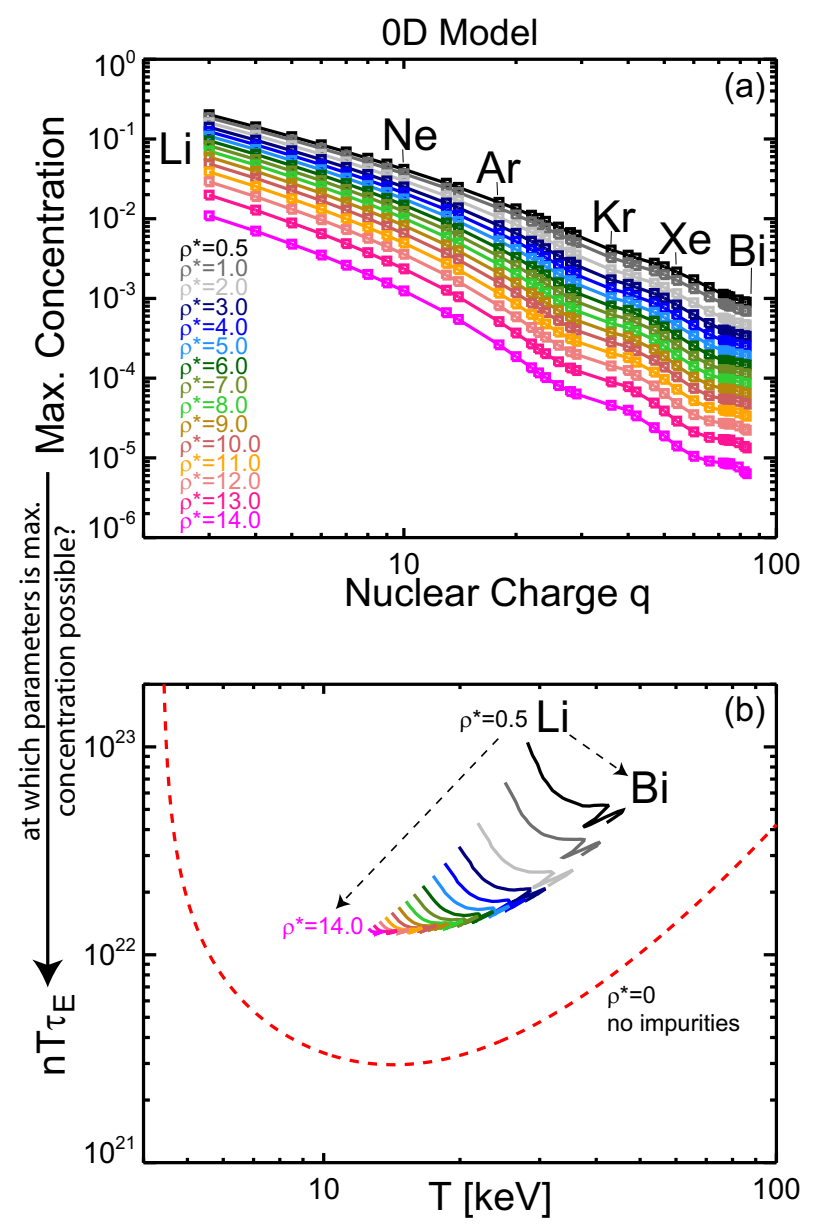

Figure 3. (colored in online-version) (a) The maximal impurity concentrations (symbols) allowing for a point-like burn curve are connected with a linear interpolation. (b) The locations of the point-like burn curves in the $n T \tau_{E}$ versus $T$ plane are given for various $\rho^{*}$ and impurities.

low-Z concentrations over tolerable high-Z concentraions is smaller than for large $\rho^{*}$. For example, at $\rho^{*}=0.5$ the limit for lithium is $20 \%$ and for bismuth it is $9.0 \cdot 10^{-4}$, which is approximately a factor of 220 different, while for $\rho^{*}=14$ this factor is approximately 1700. This can be understood, when considering figure 3(b), in which the location in the $n T \tau_{E}$ versus $T$ plane are given at which the maximum impurity level leads to a point-like burn condition. For small $\rho^{*}$, e.g. $\rho^{*}=0.5$, the difference in that location between low-Z impurities and high- $Z$ impurities is largest. This difference exists, because the largest amount of high- $Z$ impurities can be tolerated at high temperature, where radiation is reduced due to a 
smaller cooling factor, but fusion power is increased. For low-Z impurities, dilution yields the limit and thus, a lower He production and the increasing Bremsstrahlung at higher temperature moves the parameter location at which the maximal amount of low- $Z$ impurities are tolerated to lower temperatures w.r.t. the corresponding location for high-Z elements. For high values of $\rho^{*}$, e.g. $\rho^{*}=14$, the power balance is always dominated by effects due to dilution and thus, the parameter space in which most impurities can be tolerated is in a region which is better compatible with low- $Z$ impurities than with high- $Z$ impurities. Thus, the above discussed ratio increases from 220 to 1700 .

It was attempted to summarize the presented findings in a simple scaling formula, which would give the maximal impurity level $c_{Z, s c a l}$ as a power law of $Z$ and $\rho^{*}$. However, a simple power law failed, because threshold-like effects exist (no burn condition for $\rho^{*}>15.2$ ). Additionally, the $Z$-dependence is not independent of $\rho^{*}$ (cf. discussion above) and vice versa the $\rho^{*}$ dependence is not independent of $Z$. Therefore, the parameters of a more complicated formula have been fit and figure 4 results. The obtained fit formula reads:

$$
\begin{aligned}
& c_{Z, \text { scal }}=a_{0}\left(\rho^{*}\right)^{a_{1}+a_{2} Z}\left(15.2-\rho^{*}\right)^{a_{3}} Z^{a_{4}+a_{5} \rho^{*}} \quad \text { with } \\
& a_{0}=0.189547 \pm 0.0654506 \quad a_{1}=-0.0918019 \pm 0.06 .96823 \\
& a_{2}=-0.00286554 \pm 0.00135369 \quad a_{3}=0.890157 \pm 0.122136 \\
& a_{4}=-1.76106 \pm 0.0595385 \quad a_{5}=-0.0320435 \pm 0.00878319
\end{aligned}
$$

As can be seen in the given formula, the distance of $\rho^{*}$ from 15.2 is contributing to the

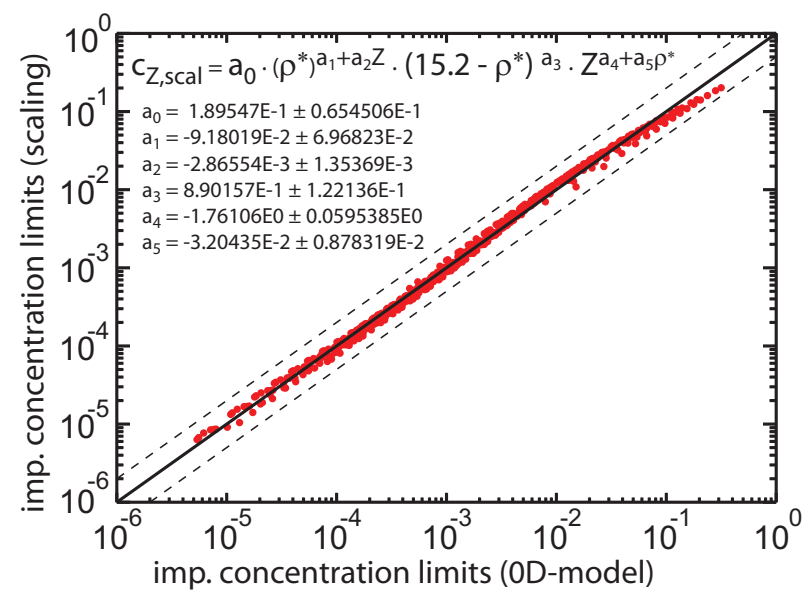

Figure 4. (colored in online-version) Comparison of the maximal impurity concentrations from the scaling formula and the actual results from the $0 \mathrm{D}$-model.

scaling independently from $\rho^{*}$ and the exponents of $\rho^{*}$ and $Z$ feature a basic dependence on the respective other parameter. The exponent of $\rho^{*}$ varies from -0.10 to -0.33 . The exponent of the term $15.2-\rho^{*}$ is slightly weaker than linear. This implies that for small $\rho^{*}$ a change in $\rho^{*}$ is relatively unimportant, while the same $\rho^{*}$ change for a $\rho^{*}$ close to 15.0 means a large relative change of the term $15.2-\rho^{*}$. A clear effect of that term in comparison to the pure $\rho^{*}$ term is relevant for $\rho^{*}>5$. So, for a reactor relevant $\rho^{*}$, i.e. $\rho^{*} \leq 5$, the correction term is less important and a relatively weak $\rho^{*}$ effect is documented. The maximum impurity concentration depends much more strongly on the impurity type, i.e. its nuclear charge $Z$. 
The exponent for $Z$ varies between -1.78 and -2.21 within the considered $\rho^{*}$ range and gives rise for the drastically different results for low- $Z$ and high- $Z$ elements.

Note that the investigated $0 \mathrm{D}$ model represents a very optimistic case, as the full plasma has constant parameters optimal for the respective impurity. At the same time no boundary conditions, like a fixed value of $n T \tau_{E}$ or a fixed, large radiated fraction have been enforced. As a first step, the next section will investigate the effect of a simplified treatment of plasma profiles.

\section{2. $0.5 D$ Power Balance with flexibility in the $n T \tau_{E} v s$. $T$ plane}

A necessary improvement of the $0 \mathrm{D}$ model seems to be the consideration of plasma profiles, because the fusion heating power has up to about $65 \mathrm{keV}$ a positive dependence on plasma temperature, while radiative losses first decrease and then increase with temperature. In a fusion plasma the edge of the plasma is colder than the core leading to a changing importance of fusion versus radiation along the plasma radius. At the same time the fusion plasma has a large volume at smaller temperatures, while the core of the plasma has a relatively small volume. In the following an approximate consideration of profiles is performed, which allows for keeping the 0D power balance scheme. The effects of profiles are taken into account via correction factors, which are calculated in the following. In order to maintain an as general validity as possible the assumptions are kept very basic. Following assumptions are introduced:

- The profiles are expressed in $x=r / a$ where the volume enclosed by $\mathrm{x}$ shall be $V(x)=L \pi a^{2} x^{2}$, i.e the plasma is mapped to a cylindrical plasma of length $\mathrm{L}$ with a circular cross-section and an outer radius $a$.

- The temperature and density profiles are linear in $x^{n}$, where $n$ is an exponent characterizing the profile shape. The profiles are characterized by their core values $T_{0}$, $n_{e, 0}$, the exponents $n_{T}$ and $n_{n}$ and the ratios $R_{T}=T_{0} /\langle T\rangle$ and $R_{n}=n_{e, 0} /\left\langle n_{e}\right\rangle$, where $\langle X\rangle$ is the volume average of the quantity $X$.

- All impurity concentration profiles are considered flat, i.e. the concentration is radially constant independently of the source location. The effects of radial impurity transport are discussed separately.

Some details and properties of the considered plasma profiles are given in Appendix A. Note, $T_{\text {edge }}, n_{\text {edge }}>0$ implies that $R_{T}<\left(n_{T}+2\right) / n_{T}$ and $R_{n}<\left(n_{n}+2\right) / n_{n}$. Moreover, hollow profiles of $T$ and $n$ are deemed unrealistic such that $R_{T}, R_{n}>1$ is a reasonable parameter space and is therefore, considered in the following.

Any plasma shape can be transformed into the cylindrical plasma described above. This may lead to unexpected differences of $T$ - and $n$-profiles when comparing the mapped to the original profiles and not always the mapped profiles are an intuitive result. Therefore, plasma elongation, which causes an obvious increase in plasma volume for unaffected midplane profiles, is treated in a special way. Specifically, the radial weighting of various effects is affected by elongation while the $T$ - and $n$ - profile shapes can be compared to those of a reactor design, which typically gives the profiles at the midplane: The elongation at the plasma edge $\kappa$ will increase the volume of the plasma at the edge, while typically the elongation towards the core of the plasma approches 1.0 corresponding to a cylindrical plasma. Thus, $\kappa$ may change the weighting of edge and core plasma, and thus, for a given $T_{0}, n_{0}, R_{n}$ and $R_{T}$, the values of $T_{\text {edge }}$ and $n_{\text {edge }}$ depend on $\kappa$. This treatment of elongation would change above equations, but as this special treatment is mostly used to address a very specific reactor design - which is discussed below - these complications are not discussed further here. Concerning 
the impurity limits the effect of elongation is minor, however, for other parameters discussed below it makes a notable difference. Below an elongation profile with linear dependence on $r / a$ ranging from 1.0 in the plasma core to $\kappa$ at the plasma edge is considered, when indicated. In figure 5, some example profile shapes are depicted with peaking factors $R_{n / T}$ ranging from 1.3 to 3.0, while the shape defining exponent $n_{n_{T}}$ is varied in a wide range - its upper border is given by the necessity to obtain positive values up to the edge. Note that the solid lines are for circular plasmas $(\kappa=1.0)$ and the dashed lines correspond to elongated plasmas with an edge elongation of $\kappa=1.6$.
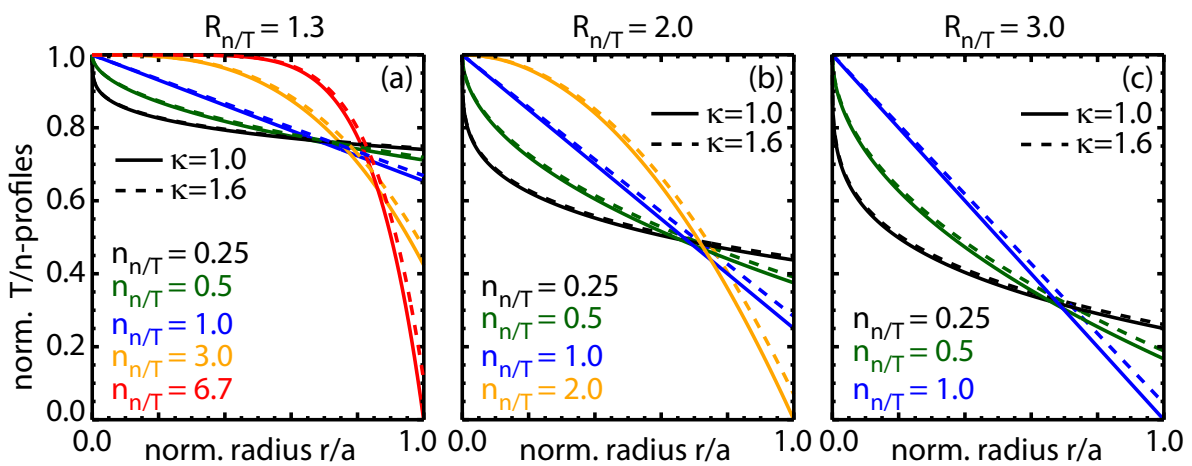

Figure 5. (colored in online-version) Normalized profiles obtained by the variation of the profile peaking factor $R_{n / T}$ and the shape defining exponent $n_{n / T}$. Profiles feature in part (a) $R_{n / T}=1.3$, in part (b) $R_{n / T}=2.0$, and in part (c) $R_{n / T}=3.0$.

For the OD power balance the power densities of $\alpha$-heating, radiation cooling and transport losses were specified. This OD scheme can be extended to considering profiles by evaluating the volume integrals of these quantities and then normalizing them with the total plasma volume. These normalized integrals resemble effective power densities. In Appendix $\mathrm{A}$, it is demonstrated that these effective power densities can be obtained with high accuracy from the 0D power densities via correction factors only dependent on $T_{0}, R_{T}, n_{T}, R_{n}$ and $n_{n}$ and not $n_{0}$. Note, that the use of profiles linear in $x^{n}$ is an unnecessary restriction, as it is shown that all considerations are independent of the size $a$ as long as $T$ - and $n$-profiles are used, for which the absolute densities and temperatures values are separated from the radial dependence, which must be expressed in terms of $x$. However, certain types of profile shapes cause the relation between $T_{0}, T_{\text {edge }}$ and $\langle T\rangle$ to be implicit which would lead to complications beyond the scope of this investigation. Thus, for simplicity here only profiles linear in $x^{n}$ are considered.

As a first step, the $0 \mathrm{D}$ results are compared for linear profiles, i.e. $n_{T}=1.0$ and $n_{n}=1.0$ which are conservatively peaked approximately matching the EU DEMO1 2015 [10] design, which will be investigated below in more detail. The temperature and density peaking factors are $R_{T}=2.1, R_{n}=1.3$. In figure 6 , the burn curves for the 0D case (cf. figure 2) considering $\mathrm{Xe}$ (grey) are compared to the $0.5 \mathrm{D}$ case (blue), while no auxiliary heating and no synchrotron losses are considered yet. Note that when experimental results are plotted in the $n T \tau_{E}$ versus $T$ plane, typically $T_{0}$ is used instead of $\langle T\rangle$, however, here the comparison to the OD model is best performed when using $\langle T\rangle$. A transformation to $T_{0}$ is straightforward as the curves are calculated for a specific $R_{T}=2.1$. When using $\langle T\rangle$, the point at which the maximum $\mathrm{Xe}$ level is tolerated almost matches that of the OD model. Still, the maximum tolerable Xe-concentration is about $15 \%$ less for the $0.5 \mathrm{D}$ case, as the 'ideal' temperature is matched 


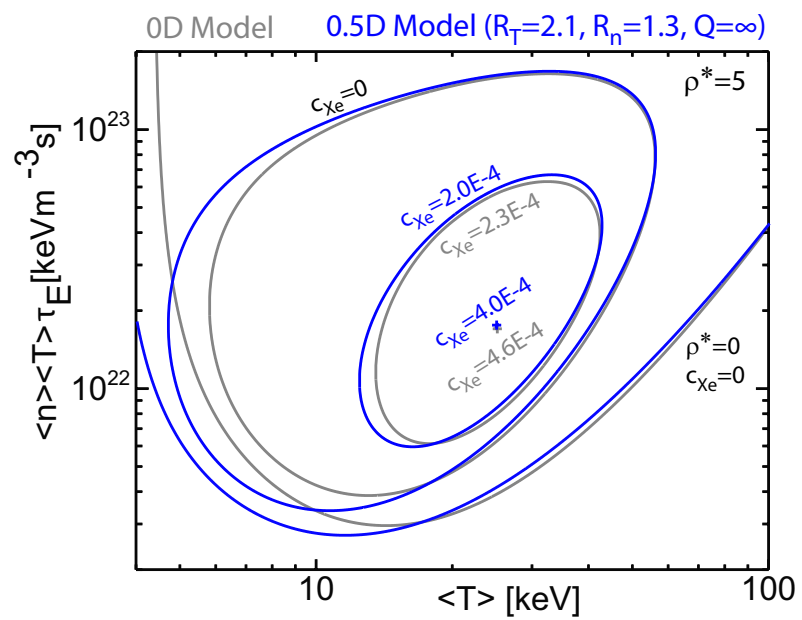

Figure 6. (colored in online-version) Comparing the burn curves for Xe for the OD-model and the $0.5 \mathrm{D}$-model assuming $R_{T}=2.1, R_{n}=1.3$ and $n_{T}=1.0, n_{n}=1.0$.

only in some part of the plasma. Comparing the burn curves at temperatures below about $15 \mathrm{keV}$ a considerable difference is observed, stemming from the fact that in the $0.5 \mathrm{D}$ case a hotter plasma core generates much more fusion power due to the strong increase of the fusion reaction coefficient in this temperature range.

When investigating the effects of profiles for all impurities and for various $\mathrm{He}$ confinement times, this boost of fusion reactions is relevant especially, when large dilution via the He ash (or via low-Z impurities) is a major player. Then the point in the $n T \tau_{E}$ versus $T$ plane where the maximum amount of impurities are tolerated is at low temperatures (cf. to the results of the $0 \mathrm{D}$ model, especially to figure $3(\mathrm{~b})$ ), i.e. $\approx 15 \mathrm{keV}$ and below, where the consideration of a peaked temperature profile leads to more than a linear boost of the fusion power in the plasma core. At the same time the radiation from low-Z elements is not a big player at these low temperatures. For large amounts of $\mathrm{He}$, the radiation from high$\mathrm{Z}$ elements is also a minor player in the power balance such that the boost of fusion power is the only major change in the power balance. Ultimately this leads to a burn at higher levels of impurities other than He as can be seen in figure 7(a) and (b) for high $\rho^{*}$ values, i.e. $\rho^{*}=10$ and $\rho^{*}=13$. In figure 7(a) the maximum tolerable impurity concentrations for flat temperature and density profiles (solid lines) are compared to those evaluated for strong temperature peaking and a flat density profile (dashed) and those evaluated for strongly peaked temperature and density profiles (short dashed). In figure 7(b) the ratio of these curves w.r.t. to the $0 \mathrm{D}$ results, i.e. the flat profiles, are plotted. Note, for all cases linear profiles are used, i.e. $n_{n}=n_{T}=1$. For the reasons explained above, the dashed and dottet lines corresponding to peaked profiles temperature profiles are above the solid lines for $\rho^{*}=10$ and $\rho^{*}=13$. The dotted lines even more so, because the additional density peaking puts even more emphasis on the core plasma region boosting fusion power further. In principle, this effect allows for a theoretical burn condition for $\rho^{*}$ values even above 19, however, it may be doubted that a fusion reactor is economically feasible if dilution is dominating the power balance, as this prevents fusion reactions, while at the same time a large reactor device is necessary to obtain enough energy confinement. A more detailed consideration of the effect of dilution on the operational space of a reactor is performed in the next section. 


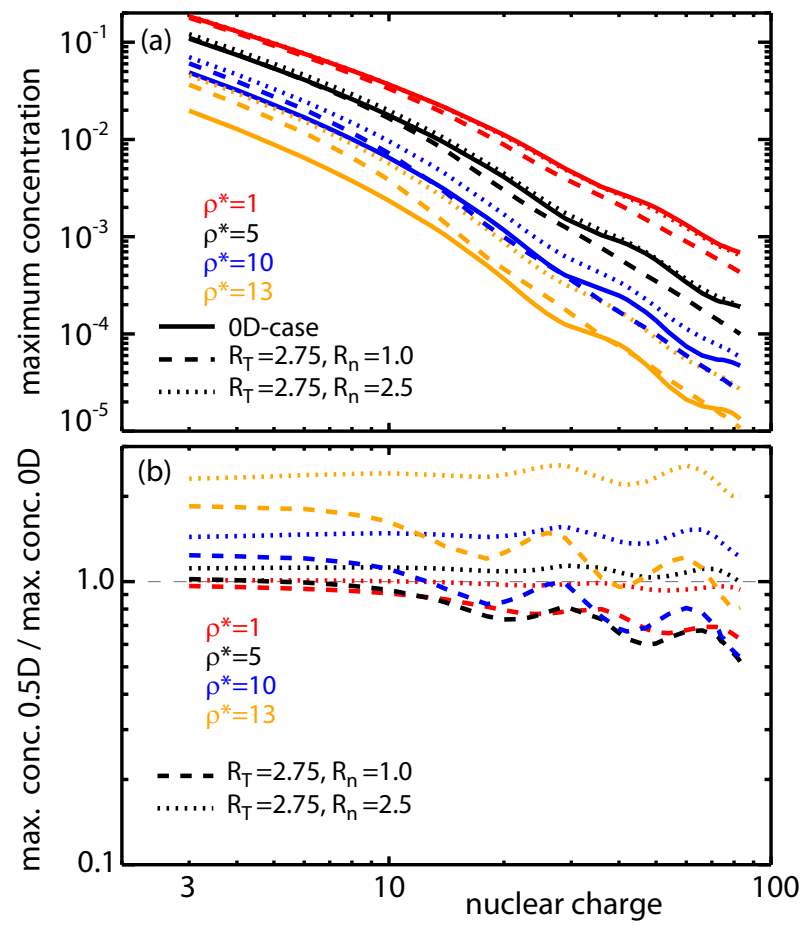

Figure 7. (colored in online-version) (a) Maximum tolerable impurity concentrations for all considered elements are given for various profile peaking parameters $R_{T}=T_{0} /\langle T\rangle$ and $R_{n}=n_{e, 0} /\left\langle n_{e}\right\rangle$ and $\rho^{*}=\tau_{H e}^{*} / \tau_{E}$. (b) Same as (a), but normalized to the respective values for flat profiles, i.e. the $0 \mathrm{D}$ case.

As a further observation, the concentrations from the $0.5 \mathrm{D}$ model feature less structure than the OD data (cf. figure 7(a)), i.e. the lines exhibit less strong curvature changes, because the radiation characteristics of each element is now smoothed due to the simultaneous presence of a wider temperature range in the plasma. For the lower values of $\rho^{*}$, i.e. lower He concentrations, the temperature profiles lead to a lower amount of tolerable impurity densities, because for low $\mathrm{He}$ concentrations the mid- $\mathrm{Z}$ to high- $\mathrm{Z}$ impurity radiation becomes a major player in the power balance. In this case, the temperature peaking leads to the occurrence of a colder, outer plasma region in which the impurity radiation from mid- $Z$ and high- $Z$ elements is increased due to the characteristics of the cooling factors. This effect is enhanced, because these cold regions are associated with a relatively large plasma volume w.r.t. to the core volume in which the fusion power is increased due to the temperature peaking. For mid- $Z$ to high- $Z$ elements this means that temperature peaking leads to even more radiative losses than additional fusion power. For low- $Z$ elements a slight gain of the fusion power over radiative power remains for $\rho^{*}=5$ due to the aforementioned reasons, an effect which disappears for $\rho^{*}=1$. Note, that when comparing the peaking and $\rho^{*}$ values to actual reactor designs, the peaking and highest $\rho^{*}$ values as presented in figure 7 are probably exaggerated. For the EU DEMO1 2015 design [10], values of $R_{T}=2.1$ and $R_{n}=1.3$ are used and the He concentration is in the range of $10 \%$ corresponding to $\rho^{*}$ smaller than $\approx 5$. When focussing on low to moderate density peaking, i.e. $R_{n}<2$, and $\rho^{*} \leq 7$, all results of the $0.5 \mathrm{D}$ model are within a factor of 2 of the results from the $0 \mathrm{D}$ model. 
In order to investigate the influence of the transport losses described by $\tau_{E}$ one can focus on cases with a flat temperature profile and a peaked density profile. In such a case the radiation and heating terms change with the square of the local density, while the transport losses only linearly change with the integral of the density. Thus, relatively the energy losses decrease. Simultaneously, the He exhaust also increases only linearly with the density integral, while the He production scales with the square of the local density. Both effects partially compensate each other such that the tolerable impurity concentration for the mentioned profiles are affected only by less than $3 \%$ (w.r.t. to the $0 \mathrm{D}$ results) for $\rho^{*} \leq 13$, $R_{T}=1.0$ and $R_{n} \leq 2.5$.

Finally the effect of the profile shape is also investigated. In figure 8, the effects of the coefficients $n_{T}$ and $n_{n}$ (cf. above and figure 5) are investigated for peaked temperature $\left(R_{T}=2.0\right)$ and peaked density profiles $\left(R_{n}=1.3\right.$ and $\left.R_{n}=2.0\right)$. The values were chosen for the clarity of the presentation, as a larger $R_{T}$ would have limited the maximal $n_{T}$. Along the same lines $n_{n}$ and $n_{T}$ are chosen in order to have examples for convex (2nd derivative is positive) and concave (2nd derivative is negative) profiles with simple numerical representation. All results are normalized to the results with flat temperature and density profiles, i.e. the $0 \mathrm{D}$ results. When studying figure $5(\mathrm{~b})$, a temperature profile with $R_{T}=2.0$

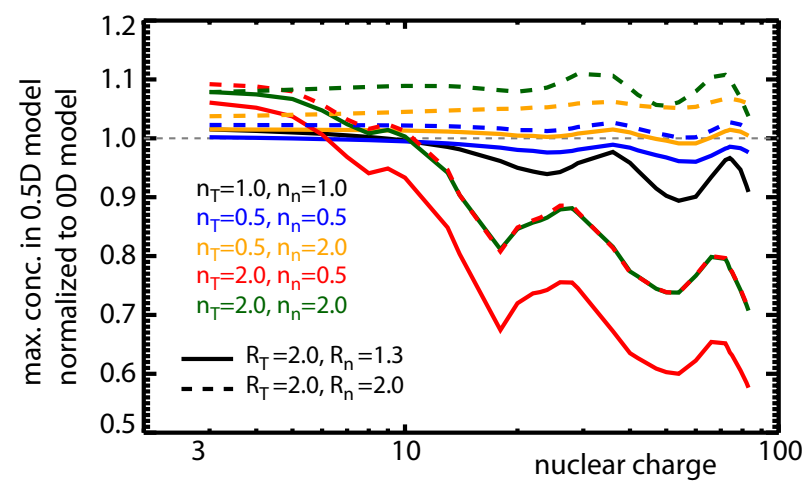

Figure 8. (colored in online-version) Maximum tolerable impurity concentrations normalized to the results from the $0 \mathrm{D}$ case for two combinations of temperature and density peaking $\left(R_{T}=2.0, R_{n}=1.3\right.$ and $\left.R_{T}=2.0, R_{n}=2.0\right)$, while the profile shapes are varied.

and $n_{T}=0.5$ leads to a small region in the plasma in which the peaking becomes important, while up to the edge considerable temperatures exist. For $R_{T}=2.0$ and $n_{T}=2.0$ the volume with larger $\mathrm{T}$ becomes clearly larger, but in the edge region very low temperatures exist, which will not contribute to plasma heating via fusion, but will increase radiative cooling. Therefore, it is understandable, that for $R_{T}=2.0$ and $n_{T}=0.5$ the $0 \mathrm{D}$ results are matched within $10 \%$, but for $R_{T}=2.0$ and $n_{T}=2.0$ larger effects are observed such that for all impurities with a nuclear charge larger than that of Ar the tolerable impurity concentration is almost a factor of 2 less than in the $0 \mathrm{D}$ case. This effect may be compensated by strong density peaking (dashed lines), which again shifts the emphasis of the profile effects towards higher temperatures. It is worthwhile to note that typical temperature profiles from today's experiments or from calculations of reactor profiles have typcially a convex shape as the normalized temperature gradient $\nabla T / T$ is not changing by much across the plasma radius. For the density profiles also concave shapes seem possible (e.g. [24]). Anyway, as the tolerable impurity concentration is mostly unchanged as long as the temperature profile is convex the impact of the profile shape for realistic plasmas seems to be limited. Note, that for the design of a fusion reactor the 
profile shapes are of large importance as they directly affect the produced fusion power.

\subsection{5D Power Balance Imposing Realistic Requirements}

Up to now the present work considered the $n T \tau_{E}$ versus $T$ plane freely accessible for a reactor, as if a reactor could choose where to operate. However, a realistic approach would be to take further requirements into account. In the following realistic boundary conditions are considered also leading to a less general validity of the model.

3.3.1. Consider Auxiliary Heating and Synchrotron Radiation Most reactor designs and also ITER do not assume to deliver all plasma heating from fusion alphas, but a majority. The ratio of total fusion power $P_{\text {fus,total }}$ including the power in the neutrons over the auxiliary heating $P_{a u x}$ gives the socalled fusion yield $Q$. For ITER values of $Q=10$ are envisaged and for reactor designs values of $Q>30$ are typical. Note, that the plasma heating by $\alpha$-particles $P_{f u s}=P_{f u s, t o t a l} / 5$ such that $P_{a u x}$ becomes a non-negligible player in the power balance of the plasma. As $Q$ relates $P_{a u x}$ rigidly to $P_{f u s}$, finite $Q$ values can be treated with the presented power balance model. To that end, $P_{f u s}$ is in the model replaced by

$$
P_{f u s, e f f}=P_{f u s}+P_{a u x}=P_{f u s}+\frac{5 P_{\text {fus }}}{Q}=P_{\text {fus }}\left(\frac{Q+5}{Q}\right),
$$

which gives for no auxiliary heating, i.e. $Q=\infty, P_{\text {fus,eff }}=P_{\text {fus }}$. In figure 9 , the burn curves for a specific case with varying $Q$ are presented. The curves correspond to the $0.5 \mathrm{D}$ model for Xe with the peaking factors $R_{T}=2.1$ and $R_{n}=1.3$, the shape coefficients $n_{n}=n_{T}=1$ and $\rho^{*}=5$. Note, that for $Q=\infty$ (blue) the curves correspond to those in figure 6, but with changed axis labels, i.e. $T_{0}$ is used instead of $\langle T\rangle$. The additional auxiliary

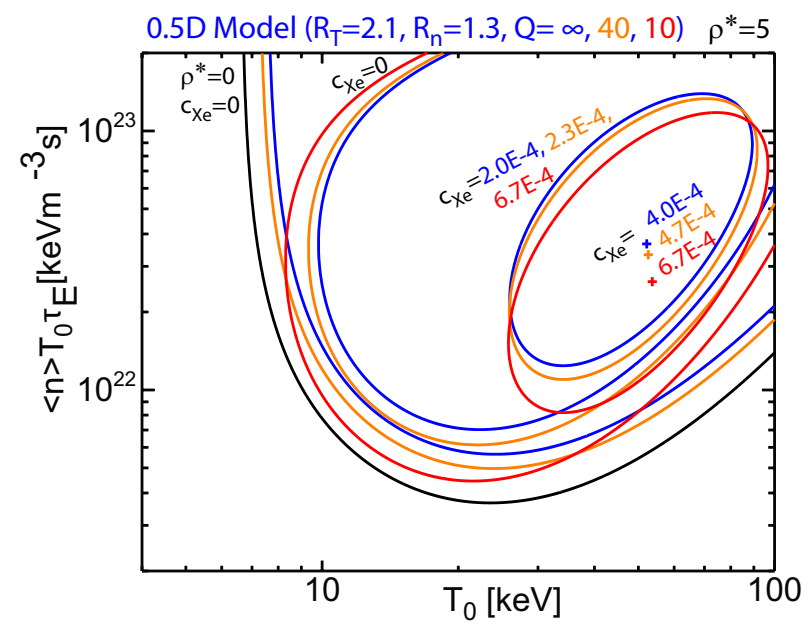

Figure 9. (colored in online-version) Comparing the burn curves for $\mathrm{Xe}$ from the $0.5 \mathrm{D}$-model assuming a fusion yield $\mathrm{Q}=\infty$ (blue), $\mathrm{Q}=40$ (orange) and $\mathrm{Q}=10$ (red).

heating leads to a larger maximum Xe concentration, while in all cases the burn curves are moved to lower $n T \tau_{E}$ values, which corresponds to lower requirements on confinement.

Similarly, additional loss power mechanisms can be accounted for by moving from $Q$ to a net fusion yield $Q_{\text {net }}$ via allowing for negative contributions to $P_{a u x}$. This feature supports 
also negative $P_{a u x}$-values, i.e. negative $Q_{n e t}$-values. This can be consistently handled down to $Q_{n e t}=-5$ at which point the additional losses are larger than the alpha heating and no burn condition may be found. Such a loss mechanism may be synchrotron radiation $\left(P_{\text {sync }}\right)$, which cannot be easily handled in the general form of the model as $P_{\text {sync }}$ strongly depends on the magnetic field [19] and major radius of the plasma and thus, on the detailed design of the reactor device. Still, for a known design the difference of auxiliary heating to synchrotron losses $P_{\text {sync }}$ is known and may be related to the fusion power via $Q_{\text {net }}$.

3.3.2. Enable Power Exhaust via Radiative Cooling Another approach to make the model more realistic is taking into account the basic needs for power exhaust. It is widely accepted that a major part of the heat flux from the plasma core needs to be reduced via impurity radiation before dedicated measures like advanced divertor designs, which handle the remaining heat flux, come into play. This means that the fraction of transported over radiated power has an upper limit. For simplicity we will assume that at least $50 \%$ of the power flux must be radiated, which is the right order of magnitude. This fraction is the socalled radiated fraction

$$
f_{\text {rad }}=\frac{P_{\text {rad }}}{P_{\text {rad }}+P_{\text {transp }}}=\frac{1}{1+P_{\text {transp }} / P_{\text {rad }}}
$$

and can be easily evaluated at each point at which a burn condition exists in the $n T \tau_{E}$ versus $T$ plane, as

$$
\begin{aligned}
\frac{P_{\text {transp }}}{P_{\text {rad }}} & =\frac{\frac{3}{2} k\langle T\rangle\left\langle n_{e}\right\rangle c_{\text {tot }} F_{\text {transport }} / \tau_{E}}{F_{\text {rad }, H} c_{D+T}\left\langle n_{e}\right\rangle^{2} L_{H}+F_{\text {rad }, H e} c_{H e}\left\langle n_{e}\right\rangle^{2} L_{H e}+F_{r a d, Z} c_{Z}\left\langle n_{e}\right\rangle^{2} L_{Z}}= \\
& =\frac{\frac{3}{2} k\langle T\rangle^{2} c_{t o t}}{\left\langle n_{e}\right\rangle\langle T\rangle \tau_{E}} \frac{F_{\text {transport }}}{F_{r a d, H} c_{D+T} L_{H}+F_{\text {rad }, H e} c_{H e} L_{H e}+F_{r a d, Z} c_{Z} L_{Z}}
\end{aligned}
$$

Thus, regions in which $f_{\text {rad }}>0.5$ is fullfilled can be easily highlighted in the $n T \tau_{E}$ versus $T$ plane. In figure 10, the burn curves for Xe in a reactor device with $R_{T}=2.1$ and $R_{n}=1.3$, $n_{T}=2.0, n_{n}=2.0, \rho^{*}=3.7, \kappa=1.6$ and $Q_{n e t}=85$, i.e. $Q=40.74 \approx 41$, $P_{\text {fus }}=2037 \mathrm{MW} \approx 2050 \mathrm{MW}, P_{\text {aux }}=50 \mathrm{MW}$ and $P_{\text {sync }}=26 \mathrm{MW}$, are depicted. These parameters are also used below, when investigating and matching the specific reactor design EU DEMO1 of 2015 [10]. Note, that from this point on a plasma elongation $\kappa=1.6$ is implemented. Underneath the burn curves $f_{\text {rad }}$ is indicated via the color-coding. Note, that the curves of constant $f_{\text {rad }}$, in particular $f_{\text {rad }}=50 \%$ run almost perpendicular to the curves indicating constant Xe-concentration. This indicates that along the $f_{\text {rad }}=50 \%$ the fusion power increases about proportionally to the Xe concentration. This is a result of the interplay between dilution, reducing the total power, and an increase of radiated power. However, for larger $n T \tau_{E}$ values $f_{\text {rad }}$ increases strongly. This is caused by an increase of dilution into that direction (cf. to next section) while the radiative power stays about constant, which means the transported fraction must be smaller for increasing $n T \tau_{E}$.

3.3.3. Avoid Strong Dilution Additionally, it is clear that strong dilution from both $\mathrm{He}$ ash or from other impurities will strongly hamper the economical feasibility of a fusion power plant. In the following we will apply a dilution criterium assuming that the created fusion power should not be reduced by a factor of 2 via dilution, which means that the sum of $\mathrm{D}$ and $\mathrm{T}$ concentrations must be more than $\approx 71 \%$. In figure 11 (a) the burn curves for Xe corresponding to the same model parameters as those in figure 10 are depicted. Additionally, 


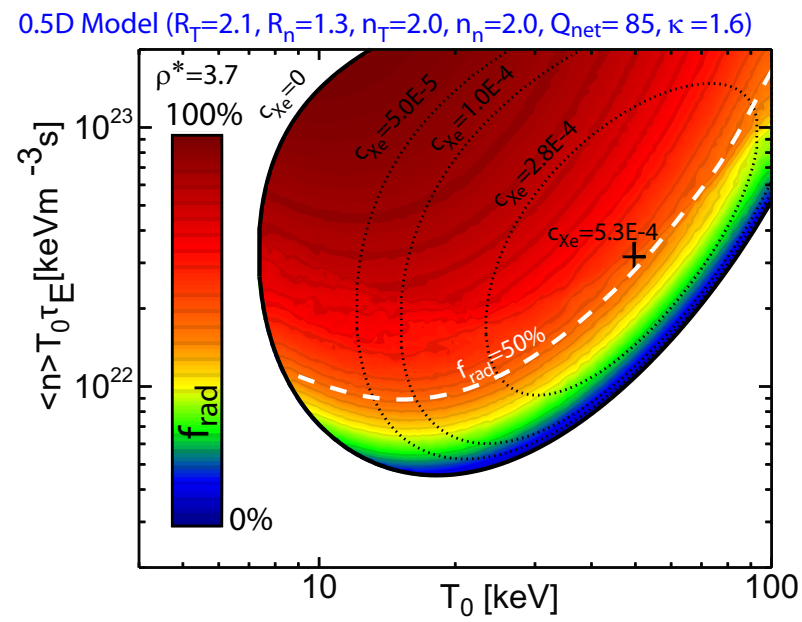

Figure 10. (colored in online-version) Burn curves for Xe are combined with a color coded map of the radiated fraction $f_{\text {rad }}$. The profile parameters and $Q_{n e t}=85$ are chosen to match the EU DEMO1 2015 design including auxiliary heating $\left(P_{a u x}=50 \mathrm{MW}\right)$ and synchrotron losses $\left(P_{\text {sync }}=26 \mathrm{MW}\right)$.

the color map indicates the corresponding He concentrations. The larger $n T \tau_{E}$ the better is the He confinement and the larger is the He production. The white dashed line labelled 'y' indicates $c_{H e}=0.145$, which therefore indicated the limit at which $c_{D+T}<0.71$ due to He dilution alone. The white dashed line 'x' corresponds to the condition $f_{\text {rad }}=0.5$ as discussed above. Both lines are drawn up to their intersection point framing a possible operational window in the $n T \tau_{E}$ versus $T$ plane. In figure 11(b), the burn curves and model are still the same, but the color map indicates $c_{D+T}$, thus, it is possible to judge the total dilution, i.e. dilution by $\mathrm{He}$ and Xe. The black dashed line ' $z$ ' which also was inserted in part (a) of the figure, indicates $c_{D+T}=0.71$. At the edge of the color map no Xe dilutes the plasma, thus, the lines ' $y$ ' and ' $z$ ' overlay. For increasing Xe concentration, i.e. towards the black cross, dilution due to Xe makes the lines ' $y$ ' and ' $z$ ' diverge, defining a smaller operational window in the $n T \tau_{E}$ versus $T$ plane. The decrease of the operation window is rather mild, as is for all high-Z elements. It may be argued, whether the critera $c_{D+T}>0.71$ and $f_{\text {rad }}=50 \%$ are too strict or too lose, however, the implications of a changed value for these limits can be judged in the example presented in figure 11. Note, that for the EU DEMO1 2015 design using Xe and $\mathrm{W}$ as core radiators, $c_{D+T} \approx 0.78$ and $f_{\text {rad }} \approx 58 \%$ with $f_{\text {rad }}$ excluding $P_{\text {sync }}$.

This operation window may be studied for various impurities, while the qualitative difference between low- $\mathrm{Z}$ and high- $\mathrm{Z}$ impurities is of particular interest. In figure 12, the effect of the operational window for $\mathrm{N}, \mathrm{Ar}$ and $\mathrm{W}$ as independent impurity is studied. As a reference case, the profile parameters, $\rho^{*}$ and $Q_{n e t}$ values of figures 10 and 11 are used and depicted as solid lines in figure 12(a). The blue $(\mathrm{N})$, orange (Ar) and red (W), solid lines frame the operational windows for the corresponding impurities. The maximum possible impurity density in the whole plane (cf. to section 3.2) for each impurity is given in the box with the solid frame, and the point in the $n T \tau_{E}$ versus $T$ plane is indicated with a coloured, solid cross. For low-Z impurities the dilution becomes a severe limitation, as for nitrogen $(\mathrm{N})$ the operation window becomes limited to relatively small temperatures and at the same time the 

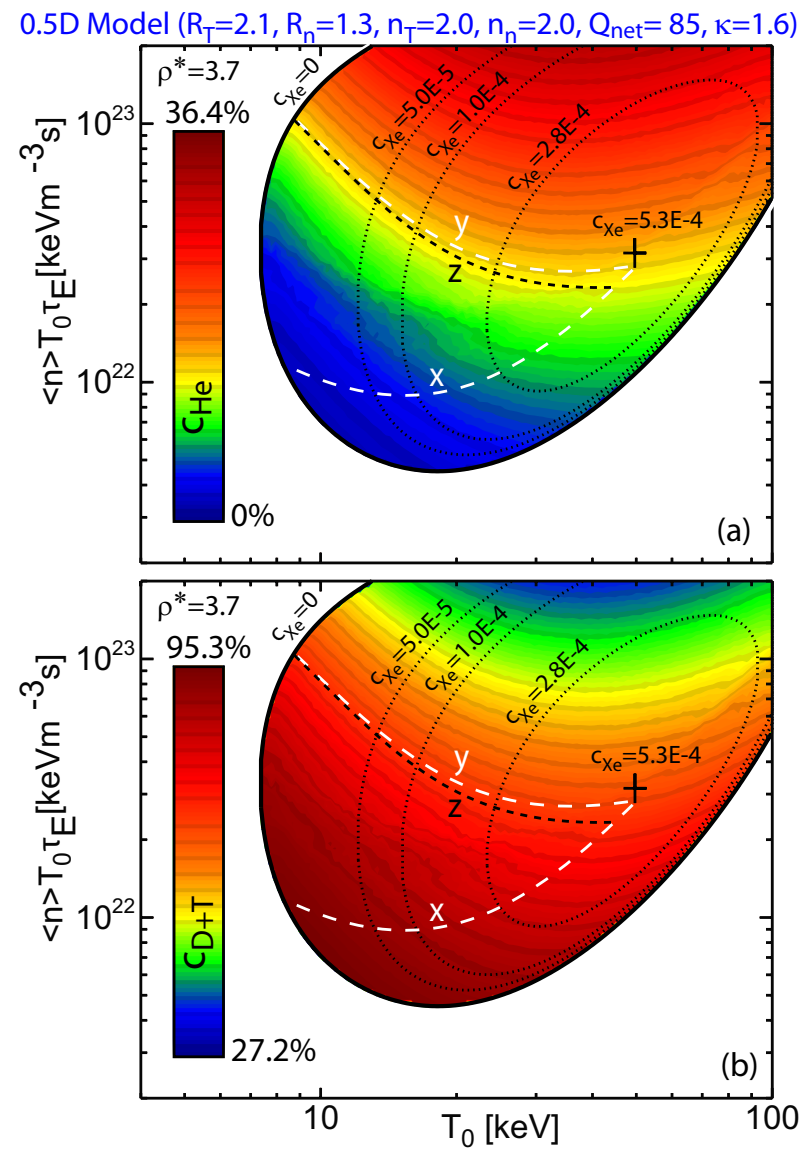

Figure 11. (colored in online-version) (a)Burn curves for Xe are combined with a color coded map of He concentrations. The meaning of the lines ' $x$ ', ' $y$ ' and ' $z$ ' are given in the text. (b) Same as (a), but the color coding gives $c_{D+T}$ thus, demonstrating all dilution effects.

demand on $n T \tau_{E}$ also increases w.r.t. to the operation window for $\mathrm{W}$, which causes negligible dilution. For Ar, the operation window is only mildly reduced as compared to that of $\mathrm{W}$ and compared to the strong reduction for $\mathrm{N}$, even though the nuclear charge of $\mathrm{Ar}$ is almost a factor of 4 smaller than that of $\mathrm{W}$. This is related to the relative large radiative cooling by $\mathrm{Ar}$ as compared to $\mathrm{N}$ limiting the absolute Ar concentration. Taking the operational point of the EU DEMO1 2015 design as a fixed point indicated as a black circle, it is inside the operational window for $\mathrm{W}$ and marginal outside that of $\mathrm{Ar}$, while the operation window for $\mathrm{N}$ is far off. For this point, $\rho^{*}=3.7$ was chosen, because for the He concentration $\left(c_{H e}=10 \%\right)$ of the EU DEMO1 2015 design (at the corresponding point in the $n T \tau_{E}$ versus $T$ plane) is matched when considering radiators that do not considerably dilute the plasma such as Xe and W. Note, that we omitted Xe in figure 12, as its operation window is almost identical to that of W.

In order to investigate if sacrifices in $Q_{n e t}$ could considerably alleviate this situation, all calculations have been also performed at $Q_{n e t}=42.5$ corresponding to either half the fusion power or auxiliary heating of $74 \mathrm{MW}$ instead of $50 \mathrm{MW}$. The corresponding results have been introduced in figure 12(a) as dashed lines and exhibit only a very mild change not changing 
the overall picture. Finally, in figure 12(b) the $Q_{n e t}=85$ of the reference case has been considered, while a much improved He exhaust using $\rho^{*}=2$ is applied. Even though the operation windows are clearly enlarged, the operation window for $\mathrm{N}$ is not including the EU DEMO1 2015 operation point.

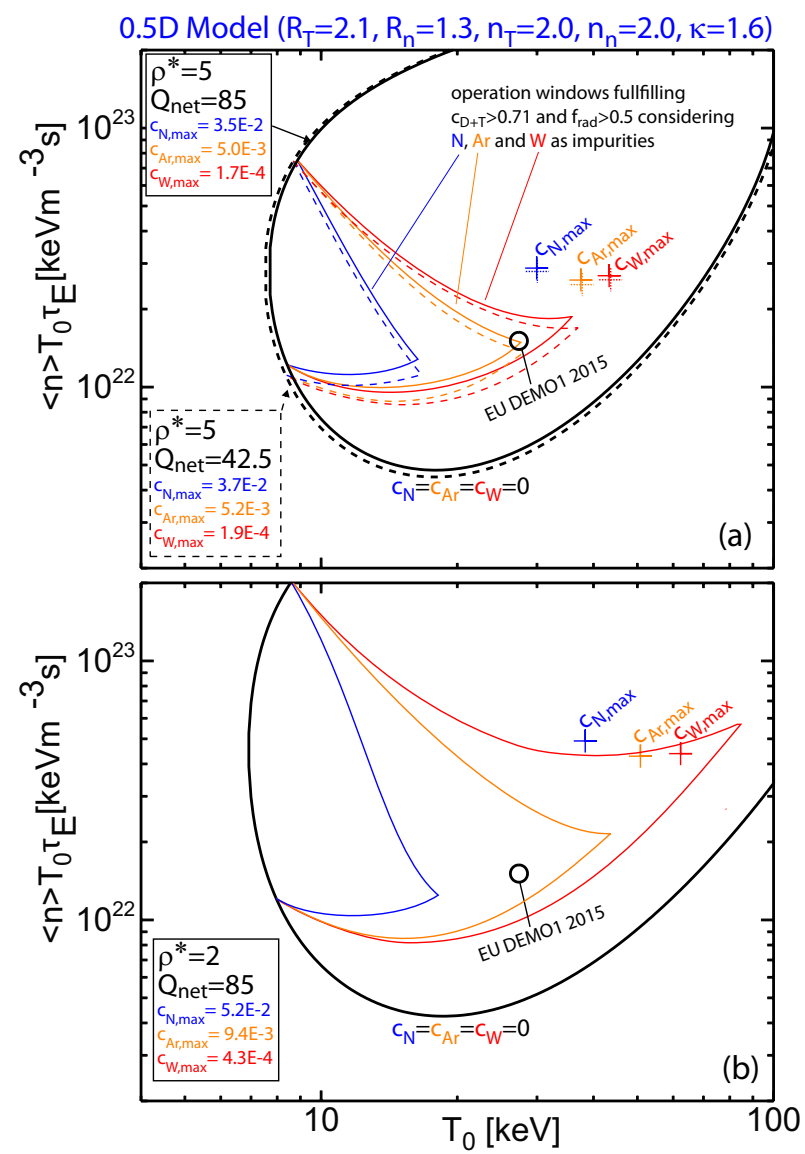

Figure 12. (colored in online-version) (a) The operation windows of a reactor device (for parameters cf. to top of figure) with $\rho^{*}=5$ are depicted for $\mathrm{N}$ (blue), Ar (orange) and $\mathrm{W}$ (red) as independent impurities. The window is limited by requiring a radiated fraction of $>50 \%$ and a dilution limit of $c_{D+T}>0.71$. The solid lines correspond to $Q_{n e t}=85$ and the dashed lines correspond to $Q_{n e t}=42.5$. (b) same as (a) but for $\rho^{*}=2$ and for only $Q_{n e t}=85$.

Note, that the above considerations are oversimplified in two aspects. First, for a reactorrelevant impurity mix it may be a good solution addressing the radiative cooling in the core and in the plasma edge separately. If separate edge radiatiors are needed this will naturally lead to edge radiators such as $\mathrm{N}$ contaminating the main plasma leading to impurity mixtures in the main plasma. Still, the idealized consideration taking only single impurities into account allows for insights, how each single impurity acts on the operation window. Second, it may be argued that the confinement of a reactor is limited by a critical plasma pressure. Thus, if dilution becomes strong there will be headroom to increase $n T \tau_{E}$, as the denoted $n$ is the electron density only, while the density of the ions and thus the plasma pressure is decreasing 
with increasing dilution. Thus, for a variation of the dilution the EU DEMO1 2015 design is a moving target in the presented plots. This complication will be addressed in section 3.4.2.

For these reasons we abstain from mapping out the the operation windows for all combinations of $n-$ and $T$-profiles and $\rho^{*}$ in order to determine the maximum impurity concentration for each element. We rather will focus on the EU DEMO1 2015 design and what impurity limits can be evaluated by our model including further improvements and a more advanced model. Nevertheless, the calculation of the operation windows in the present section demonstrates that complex design codes like PROCESS [25,26], which consider many details and which came up with the EU DEMO1 2015 design, must move within the general frame work which can be identified with the tools developed in the present work. Note, that if the operation point of the EU DEMO1 2015 design was not indicated in figure 12(a), one would still like to design a reactor in this region of the $n T \tau_{E}$ versus $T$ plane: For points in the $n T \tau_{E}$ versus $T$ plane which feature larger $\mathrm{W}$ - or Xe-impurity concentrations, i.e. points closer to the cross indicating the location of maximum impurity concentration, more fusion power is created, this is true especially when movin along the lines of constant $f_{\text {rad }}$. However, moving up in $n T \tau_{E}$ is expensive (mostly accomplished with size or magnetic field), thus, one would like to stay close to minimum $n T \tau_{E}$-values within the operation window. These considerations lead to an operational point close to the ' $x$ ' line, but right of its minimum in $n T \tau_{E}$ plane, but not too far right, as this implies increasing $n T \tau_{E}$.

\subsection{Evaluate the limit for a specific reactor design fixing $n T \tau_{E}$ and $T$}

In the following we leave general considerations on the operational space behind and focus on specific reactor designs or specific points in the $n T \tau_{E}$ versus $T$ plane. Note, that the determination of the impurity concentration and other parameters such as the He concentration are still governed by the power balance and helium balance, while the dilution and radiative fraction are not a boundary condition but rather a result of the model.

3.4.1. Assuming a fixed $Q, n T \tau_{E}$ and $T$ For a fixed point in the $n T \tau_{E}$ versus $T$ plane, a fixed He exhaust and a fixed $Q$ it is straight-foward for the $0.5 \mathrm{D}$ model to evaluate the impurity concentrations leading to a steady state burn. Generally, these impurity concentrations are clearly smaller than the maxium possible impurity concentrations evaluated in section 3.2, because the reactor design typically does not hit the point in the $n T \tau_{E}$ versus $T$ plane at which this concentration is possible. In the following, this simple evaluation will be referred to as ' $0.5 \mathrm{D}$ model with fixed $T$ and $Q$ ' and will serve as a reference to the more complicated evaluations allowing for flexibility in $T$ and $Q$.

3.4.2. Allowing for changes in $Q$ and $T$ as a consequence of dilution The reactor performance, even though important boundary conditions are fixed, may be dependent on the dilution of the plasma. Even within the operation window in which dilution is low enough to operate a reactor the $Q$-value will change depending on the exact values of the dilution. Additionally, increasing dilution is reducing the plasma pressure at constant electron density (noted as $n$ in the present work) and temperature. If the plasma pressure is considered as the only limit for plasma confinement, then dilution implies that higher $T$ values are accessible, which below $T=65 \mathrm{keV}$ leads to an increase in fusion power compensating the aforementioned loss due to a reduced fuel density. As a further consequence, more impurities might be tolerated by the plasma in steady-state.

Due to the fact, that the final impurity levels, $Q$-values and $T$-values do meet a steadystate condition, the $0.5 \mathrm{D}$ model must be able to capture this burn condition, too. However, the 
appropriate values of $Q$ and $T$ must be determined iteratively. For that purpose, we start from the design values of $Q$ (i.e. $\left.P_{f u s}\right), T, c_{D+T}$ and $c_{t o t}$ as a reference and keep them labelled $Q_{\text {ref }}, P_{\text {fus }, \text { ref }}, T_{\text {ref }}, c_{D+T, \text { ref }}$ and $c_{\text {tot,ref }}$. We take these values from the EU DEMO1 2015 design as $Q_{\text {ref }}=40.74 \approx 41, T_{\text {ref }}=27.36 \mathrm{keV} \approx 27.4 \mathrm{keV}$ and $c_{D+T, \text { ref }} \approx 0.78$. In order to obtain these reference values with the shapes as parametrized in the $0.5 \mathrm{D}$ Model, $n_{T}=n_{n}=2.0$ are chosen, for $R_{T}=2.093 \approx 2.1, R_{n}=1.27 \approx 1.3$ and $\kappa=1.6$, consistent with the EU DEMO1 2015 design. It is worthwhile to note, that $n_{T}=n_{n}=2.0$ were chosen to obtain the design value $P_{f u s}=2037 \approx 2050 \mathrm{MW}$, while matching geometry, $T_{0}, R_{T}, n_{0}, R_{n}$ and $\kappa$. Such profiles are typically too broad in comparison to profile shapes as obtained when taking realistic transport coefficients for heat and particles into account. However, in order to match the $Q$ from the PROCESS run $n_{T}=n_{n}=2.0$ was chosen. Also, $\rho^{*}=3.7$ is determined via the comparison to the PROCESS run for the EU DEMO1 2015 design, which features $10 \%$ of helium. Note, that an alternative set of reference parameters could also be obtained independently of an existing design by choosing a spot in the $n T \tau_{E}$ versus $T$ plane, a $Q$-value and a reference impurity.

In contrast to the above approaches $Q$ is now changed according to the evaluated changes in $P_{\text {fus }}$ (cf. next paragraph), while $P_{\text {aux }}$ is kept constant. The changes in $P_{\text {sync }}$ are considered negligible. Note, in figure $12 Q$ was fixed for the whole plot, which implies that $P_{a u x}$ is adjusted to the level of dilution. Now, we assume $P_{a u x}$ fixed.

In the following we assume that in a specific reactor design, i.e. the reference, $n$ is already maximized and limited by additional physics such as a density limit and thus, a change of the electron density is not envisaged. Assuming constant plasma pressure, $n T c_{t o t}$ is a constant and as $n$ is a constant $T$ changes inversely to $c_{t o t}$. Assuming that for all further considerations $P_{a u x}$ is fixed, the fusion power directly translates into $Q$. In order to evaluate the correct fusion power for constant $n$, one requires the knowledge of $c_{D+T}$ and $T$. The necessity of iterating the $0.5 \mathrm{D}$ model stems from the problem, that only after the evaluation of the burn curves in the $n T \tau_{E}$ versus $T$ plane the exact He and impurity concentrations are known, which then influence the actual $Q, T$ and $n T \tau_{E}$ values of interest. As for low-Z impurities the dilution is most important, the largest effects are expected for them, while for high-Z impurities only a minor impact of this approach is expected. One iteration step for an impurity $\mathrm{Z}$ from $k^{\text {th }}$ to the $(k+1)^{\text {st }}$ evaluation is described by the following procedure:

- Use $Q_{k}, T_{k}, n T_{k} \tau_{E}$, and run the $0.5 \mathrm{D}$ model for impurity $\mathrm{Z}$

- Determine $c_{D+T, k}$ and $c_{t o t, k}$ at $n T_{k} \tau_{E}$ versus $T_{k}$

- Determine $T_{k+1}=T_{r e f} \frac{c_{t o t, r e f}}{c_{t o t, k}}$ and $Q_{k+1}=Q_{r e f} \frac{P_{f u s}\left(c_{D+T, k}, T_{k+1}\right)}{P_{f u s, r e f}}$

- If the change in $T$ and $Q$ is smaller than $0.1 \%$ then stop iteration.

For the evaluations presented here, the convergence was never an issue, as the dilution causes only mild changes in $\mathrm{T}$, while a moderate change in $Q$ also realizes only mild changes in the in impurity concentrations (cf. figure 12(a)) and thus, dilution. Nevertheless, it is possible to judge the convergence by examining figure 13, which shows the $Q$ (cf. figure 13(a)) and $T$ (cf. figure 13(b)) values for $\mathrm{Li}, \mathrm{N}, \mathrm{Ar}, \mathrm{Kr}$ and $\mathrm{Xe}$ for all iterations until convergence. The used 0.5D model corresponds to the EU DEMO1 2015 design using $\rho^{*}=3.7\left(c_{\mathrm{He}} \approx 10 \%\right)$ for the design point. In figure 13(c) the relative change of the impurity level with respect to the first evaluation is given, and in figure 13(d) the corresponding He concentrations are depicted.

The iterative process for the design point (Xe for $\rho^{*}=3.7$ ) is very short, because the design point is the reference point and already in the converged state. For the other impurities the largest changes happen if $c_{t o t}$ obtained in the first evaluation is quite different 

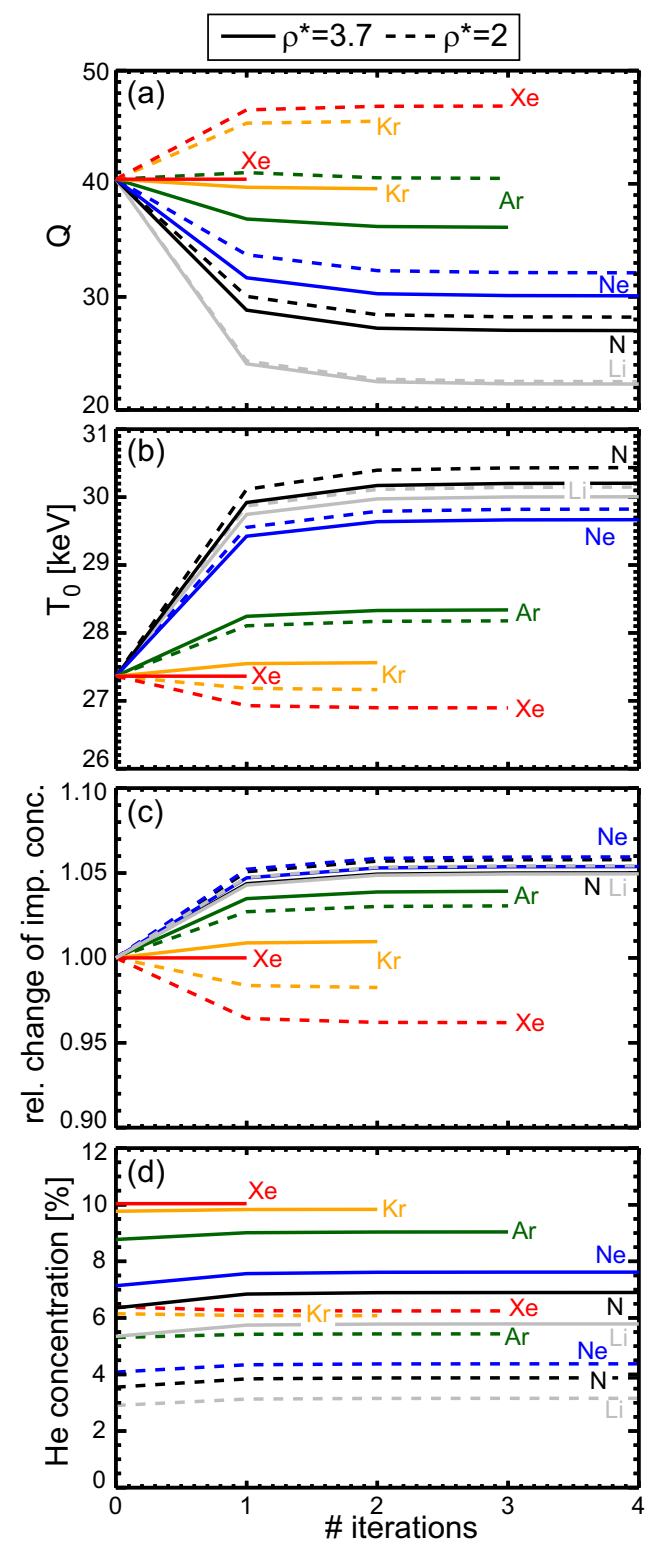

Figure 13. (colored in online-version) For the enhanced 0.5D reactor model corrections to $Q$ (due to dilution and $T$ changes) and $T$ (assuming constant plasma pressure) are evaluated iteratively. This is done for a reactor plasma with different impurities (color coded) and two values of $\rho^{*}$ (solid, dashed). (a) $Q$ is depicted, which is an input at each iteration, and, thus, at iteration ' 0 ' the reference value of 41 is used. (b) The core $T$ is depicted, which is an input at each iteration and, thus, at iteration ' 0 ' the reference value $27.4 \mathrm{keV}$ is used. (c) The relative change of the impurity concentration w.r.t. to the reference scenario is evaluated, thus, the output of iteration ' 0 ' serves as the reference. (d) The He concentration as evaluated at each iteration is depicted, thus, this is an output at each iteration.

from $c_{t o t, r e f}$, which results in an adjustment of $Q$ and $T$ for the next evaluation. For Li The 
adjustments of $Q$ are almost a factor of 2, while the temperature changes by only about $10 \%$. Convergence is obtained within at most 4 iterations for all cases.

When considering $\rho^{*}=2$, also for Xe the value $c_{t o t}$ is different from $c_{t o t, r e f}$ due to the much smaller He content, such that the assumption of constant plasma pressure leads to a reduction of the plasma temperature by about $500 \mathrm{eV}$. Nevertheless the converged $Q$ has increased as less dilution dominates the change in $Q$.

Note, that all iterations may also be performed with flexible $Q$ and fixed $T$, which then clearly reveals that the $T$ change obtained from the assumption of constant plasma pressure is only a small correction to the effects of dilution. Such a comparison is presented below. Note, that the impact of the iterative treatment with flexible $Q$ and $T$ on the obtained impurity level is rather mild as they are affected within a $6 \%$ margin only (cf. figure 13(c)). Still, the iterative approach yields the advantage that $Q$ can be calculated self-consistently for a reactor design with fixed $P_{a u x}$, while all the advantages of the $0.5 \mathrm{D}$ model prevail.

Note, that for all cases the allowed impurity concentrations are normalized to that of iteration ' 0 ' in order to visualize the relative impact of the iterative approach. So, for example the allowed Xe concentration for $\rho^{*}=2$ goes down for each iteration, but the absolute values are larger than those for $\rho^{*}=3.7$.

\subsection{Compare to a realistic ASTRA simulation of the EU DEMO1 2015 Design}

ASTRA (cf. [27, 28]) is a modelling code which can solve the radial transport equations for particles, energy and momentum. The transport coefficients can be put in, or evaluated via various models and therefore, it is a very versatile tool. The EU DEMO designs have been investigated using ASTRA earlier (e.g. [29,30]) and the possibilities to investigate the properties of such a reactor design are manifold. Ultimately, ASTRA and models within, such as TGLF [31] may be used to benchmark high-level physics models with the simple design assumptions made in PROCESS. This is not the aim of the present work, which uses ASTRA in a simplified mode, treating heating sources, radiation losses and heat transport self consistently. In detail, the heating sources and the radiation distribution is made consistent with the temperature profiles by assuming a heat transport coefficient profile as obtained from earlier investigations [30]. This profile is scaled such that $P_{\text {fus }}=2050 \mathrm{MW}$ is obtained for the design point, resulting in $T_{0} \approx 34 \mathrm{keV}$ as the temperature profiles are close to linear rather than parabolical. Note, that the ASTRA profile seems to be more realistic than the parabolic shape, nevertheless the latter was used above for the $0.5 \mathrm{D}$ model in order to match the $P_{\text {fus }}$ obtained from PROCESS, while also matching $T_{0}, R_{T}, n_{0}, R_{n}$ and $\kappa$ from PROCESS. The heat transport coefficients are then kept constant allowing an interaction between radiation, alpha heating and temperature profiles. The electron density profile in ASTRA was fixed for all cases to that used in the $0.5 \mathrm{D}$ model. For these profiles, various combinations of $\mathrm{He}$ concentrations and impurity levels for $\mathrm{N}, \mathrm{Ne}, \mathrm{Ar}, \mathrm{Kr}$, and Xe were scanned and the impact on the temperature and $P_{\text {fus }}$ was documented. The reactor relevant impurity level is then determined via a condition on the edge heat flux, i.e. it must be 1.2 times the power LH-threshold, which is $154 \mathrm{MW} \approx 150 \mathrm{MW}$ for the EU DEMO1 2015 design. The energy confinement time $\tau_{E}$ is obtained from the transported power at the edge and the stored energy and the He confinement time $\tau_{H e}^{*}$ is obtained from the He content and the fusion rate. Thus, $\rho^{*}$ is known.

This procedure results in figure 14(a) giving for $\rho^{*}=3.7$ the impurity concentrations for $\mathrm{N}, \mathrm{Ne}, \mathrm{Ar}, \mathrm{Kr}$ and $\mathrm{Xe}$ at the edge power loss of $150 \mathrm{MW}$. These values are compared to the $0 \mathrm{D}$ model (for reference) giving the maximum impurity concentration in the full $n T \tau_{E}$ versus $T$ plane (blue) and various implementations of the $0.5 \mathrm{D}$ model. The latter are all evaluated 
at the $n T \tau_{E}$ versus $T$ values relevant for the EU DEMO1 2015 design. The orange dashed line corresponds to the impurity limits at $n T \tau_{E}=1.51 \cdot 10^{22} \mathrm{keV} \mathrm{m}^{-3} \mathrm{~s}$ and $T_{0}=27.4 \mathrm{keV}$ for a fixed $Q=41$. Note, that $\tau_{E}=6.9 \mathrm{~s}$ was used in order to consider a radiation corrected $\tau_{E}$, which corresponds to the definition used in the $0 \mathrm{D}$ and $0.5 \mathrm{D}$ models. The short dashed red line indicates the impurity levels for the same $n T \tau_{E}$ and $T_{0}$ values, but at the $Q$-value as calculated from dilution only, i.e. comparing the $c_{D+T}$ value to $c_{D+T, \text { ref }}=0.78$ and the solid magenta line gives the impurity levels when dilution not only affects $Q$ but also $T$, which mildly adjusts $Q$ again. The $0.5 \mathrm{D}$ models differ among each other in their predicted impurity concentrations by less than $10 \%$ consistent to the cases investigated above and in figure 13 . In figure 14(a), it is visible that the ASTRA runs match the $0.5 \mathrm{D}$ model for $\mathrm{Xe}$ as this is the reference case for which the $0.5 \mathrm{D}$ model was matched via choosing $n_{T}, n_{n}$ and $\rho^{*}=3.7$. For ASTRA, adjustements of $T_{0}$ have been performed to match $Q$ and the He content matches as a consequence of $\rho^{*}=3.7$.

For lighter impurities than Xe, the ASTRA runs allow for notable less impurity content than the $0.5 \mathrm{D}$ model. Comparing the helium content for all models (cf. figure 14(b)), one finds the 0D model predicts a considerably higher He content as it is evaluated at larger $n T \tau_{E}$. This difference is mostly given by the different spot in the $n T \tau_{E}$ vs. $T$ plane at which the models are evaluated. For all 0.5D models and the ASTRA simulations the He content behaves remarkably similar, even though the $Q$ value is adjusted only in two of the three 0.5D models. However, the case $Q=40.74$ (orange, dashed) should be interpreted as an adjustment in $P_{a u x}$, while $P_{f u s}$ does change according to dilution. Thus, it is straightforward to understand, that with more dilution, i.e. for low-Z impurities, less He content is expected as its production decreases as does $P_{f u s}$. For the two 0.5D models for which $Q$ has been adjusted, explicitly $P_{a u x}$ is kept constant, and the behaviour of the He content moves according to the $Q$ dependence. Note, that the $0.5 \mathrm{D}$ model for which the dilution affects $Q$ and $T$ of the plasma (magenta, solid) ends up with slightly higher helium content and slightly higher $Q$ than the $0.5 \mathrm{D}$ model in which dilution only affects $Q$ (red, dashed).

It is worthwhile to focus on the small differences between the two $0.5 \mathrm{D}$ models featuring adjustments of $Q$ and the ASTRA model. In figure 14(b), ASTRA predicts slightly less helium than the 0.5D model without $T$ increase, while in figure 14(c), the ASTRA simulation follow the trend of the $0.5 \mathrm{D}$ model with $Q$ and $T$ adjustment. This subtle behaviour may only be understood fully, when looking at the $T$ changes as observed in the ASTRA modelling.

In figure 15 , the $T$ changes as in the $0.5 \mathrm{D}$ model (magenta curves) are compared to the changes of the central $T$ in the ASTRA model, by relating the changed $T$-value to the $T_{\text {ref }}$, i.e. the central temperature of the reference case. Thereby, the case with Xe and $10 \%$ of $\mathrm{He}$ serves as a reference. As in the ASTRA model $T$ changes are driven by changes in heating, the relative $T$ change in all ASTRA simulations scales with $c_{D+T}$, which is driving the production of $\alpha$-particles. The temperature changes in the $0.5 \mathrm{D}$ model are driven by dilution, thus, $c_{t o t}$. As $c_{t o t}$ scales not quite like $c_{D+T}$ for different impurity mixtures, the various $\rho^{*}$ values do not align perfectly to one line for the $0.5 \mathrm{D}$ model. When plotting $T / T_{\text {ref }}$ versus $c_{D+T}$ for model runs scanning all elements at three different $\rho^{*}$-values the three pink lines in figure 15 result. Clearly, a qualitative difference between the two models, i.e. ASTRA and the 0.5D model, becomes apparent. The $0.5 \mathrm{D}$ model gains $T$ if dilution is increased, while the transport model features a decreased temperature for smaller heating power. This behaviour of ASTRA can explain the lower helium content for low-Z impurities (cf. figure 13(b)), but is seems surprising that the ASTRA simulation follows the $Q$ dependence of the $0.5 \mathrm{D}$ model which features increasing $T$ (magenta, solid) for increasing dilution (cf. figure 13(c)).

However, the boundary condition for the ASTRA simulation is that the heat flux at the plasma edge adds up to $150 \mathrm{MW}$, independent of dilution. This implies, that for impurities 

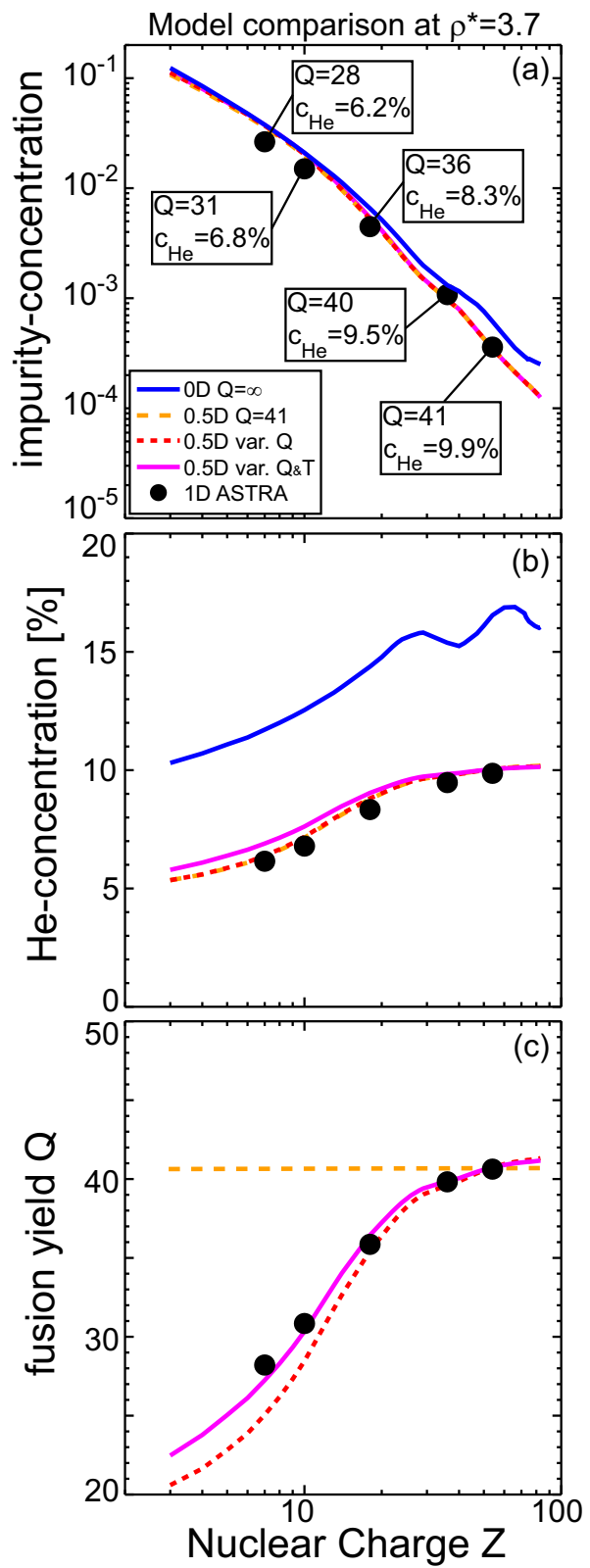

Figure 14. (colored in online-version) (a) Depicted are the obtained impurity concentrations from the 0D model, three variants of the $0.5 \mathrm{D}$ model matching the profiles of the EU DEMO1 2015 design and the 1D ASTRA simulation. One 0.5D model assumes constant $Q$, i.e. flexible $P_{a u x}$, the second 0.5D model considers the effect of dilution on $Q$, and the third considers the effect of dilution on both $Q$ and $T$. (b) For all models the corresponding He concentrations are given. (c) For all models the corresponding $Q$ values are given.

which are capable to cause strong dilution a more strict limit applies, as the tranported power stays at $150 \mathrm{MW}$ independently of the heating power. This limit to the impurity concentration 


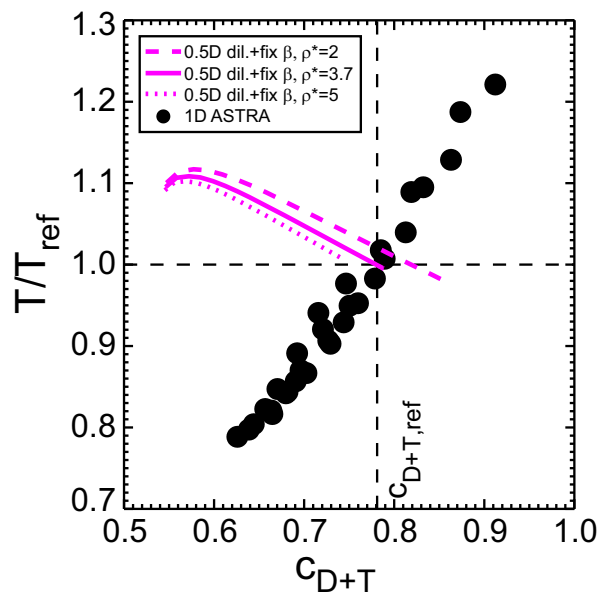

Figure 15. (colored in online-version) The relative $T$-changes for the 1D ASTRA runs are compared to those from the $0.5 \mathrm{D}$ model predictions, which assume constant plasma pressure. A clear dependence on $c_{D+T}$ is seen for the $T$-changes in the ASTRA runs demonstrating that changes in the $\alpha$-heating are driving the $T$-changes.

of low-Z impurities leads to a less strong drop of $Q$ (cf. figure 13(c)) and it explains why ASTRA features clearly lower impurity concentrations for low-Z elements (cf. figure 13(c)). Note, that both effects, i.e. the effect of dilution on $T$ and the effect of a given edge heat flux, lead to a lower tolerable impurity content than any of the proposed $0.5 \mathrm{D}$ models.

Next the dependence of the impurity concentrations, helium content and $Q$ versus $\rho^{*}$ (cf. figure 16) are investigated. At $\rho^{*}=3.7$, the behaviour as described above is obtained. For high- $Z$ impurities the all models agree well and for low- $Z$ impurities less impurity content is allowed for the ASTRA runs (cf. figure 16(a)), while the He content (cf. figure 16(b))and $Q$ (cf. figure 16(c)) show little differences as explained above. For very small $\rho^{*}$, and small dilution, i.e. for $\mathrm{Xe}$ and $\mathrm{Kr}$, an increase in tolerable impurity content is seen along with more He content and higher $Q$ indicating an increased $T$. At very large $\rho^{*}$ the dilution in ASTRA is causing a clear drop of $T$ and thus, a smaller He content and smaller $Q$ value is obtained compared to the $0.5 \mathrm{D}$ models. Especially, the slope of the ASTRA points for He concentration and $Q$ versus $\rho^{*}$ are much steeper in figure 16(b) and (c) than for either $0.5 \mathrm{D}$ model. This in turn has the effect that the allowed low-Z impurities between ASTRA and the 0.5D models agree better at large $\rho^{*}$, as the various effects compensate.

\subsection{Conclusions Drawn from the Comparisons}

The topic of impurities in a reactor device is a multifaceted topic, which cannot be comprehensively treated in one paper. In the present work, it was attempted to identify fundamental trends and systematic dependences of the physics which defines the impurity limits in a reactor. Thereby, the OD model served as a reference, which identifies the most important parameters of the impurity limit allowing for formulating a scaling law which gives an approximate impurity limit. When comparing the $0.5 \mathrm{D}$ model to the $0 \mathrm{D}$ results, it becomes apparent, that for reactor relevant values of the He confinement and profile shapes, the differences between the 0D model and the 0.5D model are only subtle. A major change in the model predictions is observed for restricting the reactor parameter space to a specific spot 
Determination of the Tolerable Impurity Concentrations in a Fusion Reactor using a Consistent Set of Cooling Factors29
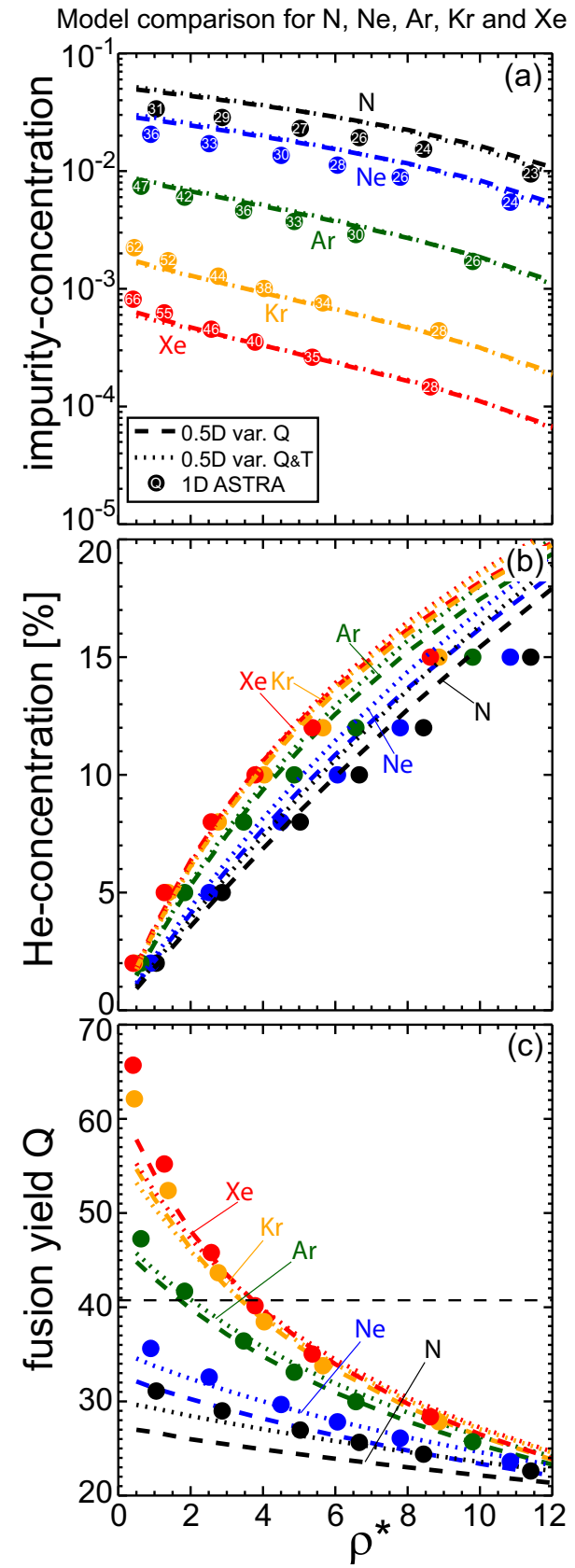

Figure 16. (colored in online-version) (a) Depicted are the obtained impurity concentrations from the two variants of the 0.5D model matching the profiles of the EU DEMO1 2015 design and the 1D ASTRA simulation. One 0.5D model considers the effect of dilution on $Q$, the other considers the effect on both $Q$ and $T$. (b) For all models the corresponding He concentrations are given. (c) For all models the corresponding $Q$ values are given. 
in the $n T \tau$ versus $T$ plane, which is a realistic consequence, when focussing on a specific reactor design. Already basic requirements on the radiative fraction and maximum dilution lead to a restriction within the $n T \tau$ versus $T$ plane underlining the aspect that not only the burn condition defines the operational window within that plane. The further extensions of the $0.5 \mathrm{D}$ model considering also changes of $Q$ and $T$ via dilution, yield subtle changes of the allowed impurity densities. As a considerable benefit, the extended $0.5 \mathrm{D}$ models can also predict the consistent He content and fusion power (and thus, $Q$ ) of a reactor device. These predictions based on a relatively simple $0.5 \mathrm{D}$ model agree with 1D ASTRA simulations quite well (within $10 \%$ ) as long as dilution is not affecting $Q$ by more than a factor of about 1.2. Even if dilution becomes important the $Q$ value and the He concentration are predicted by the $0.5 \mathrm{D}$ model within a relative uncertainty of about $10 \%$ as compared to the 1D ASTRA simulations, while the acceptable impurity density are overestimated by at most a factor of 1.5 .

When comparing the self-consistent heat transport model of ASTRA a temperature drop is observed for increasing dilution, which is trivially connected to the properties of heat transport. This is fundamentally different from an approach which foresees that for higher dilution plasma stability allows for accessing higher $T$. The latter approach assumes that transport is governed by a critical beta value or that additional heating is easily available. The comparisons performed in the present work demonstrate, that both sets of assumptions lead in the end to minor differences in the tolerable impurity content, the He content and the fusion power, because any $T$ change is small enough.

\subsection{Quantitative Results from the Iterative 0.5D Model at Various $n T \tau_{E}$ and $T$ Values}

As the iterative $0.5 \mathrm{D}$ model proved to provide realistic values for the impurity concentration, the dilution and the helium content, it is used in the following to probe the promising parameter space for a reactor. Therefore, parameter scans for all considered 33 impurities have been undertaken within the following bounds:

$$
\begin{gathered}
20 \mathrm{keV} \leq T_{0} \leq 45 \mathrm{keV} \\
1.5 \cdot 10^{22} \mathrm{keV} \mathrm{m}^{-3} \mathrm{~s} \leq\langle n\rangle T_{0} \tau_{E} \leq 2.5 \cdot 10^{22} \mathrm{keV} \mathrm{m}^{-3} \mathrm{~s} \\
2.0 \leq \rho^{*} \leq 5.0 \\
20 \leq Q_{n e t} \leq \infty \\
2.0 \leq R_{T} \leq 2.5 \\
1.3 \leq R_{n} \leq 1.6 \\
1.0 \leq n_{T} \leq 2.0 \\
1.0 \leq n_{n} \leq 2.0 \\
1.6 \leq \kappa \leq 2.0
\end{gathered}
$$

resulting in more than $10^{5}$ model reactor plasmas probing the parameter space. The scanned parameter space in $n T \tau_{E}$ versus $T$ corresponds to the region which has been identified as the most interesting region (cf. to section 3.3.2 and 3.3.3), as here dilution due to helium seems tolerable, the fusion power can be considerable and power exhaust possible. Of course, the exact outcome depends on the impurities used and the helium concentration in equilibrium. For all cases, the $0.5 \mathrm{D}$ model predicts the impurity content, the helium content and thus the dilution. For clarity, $T$ changes due to dilution and constant plasma pressure have been 
neglected, as considerations above proved that the effect is minor in any case. Additionally, the radiated fraction can also be evaluated for each case, such that for all considered model plasmas the fuel concentration $c_{D+T}$ may be plotted versus the radiated fraction (cf. figure 17). In figure 17, all model runs are depicted with the black dots and are replotted in all

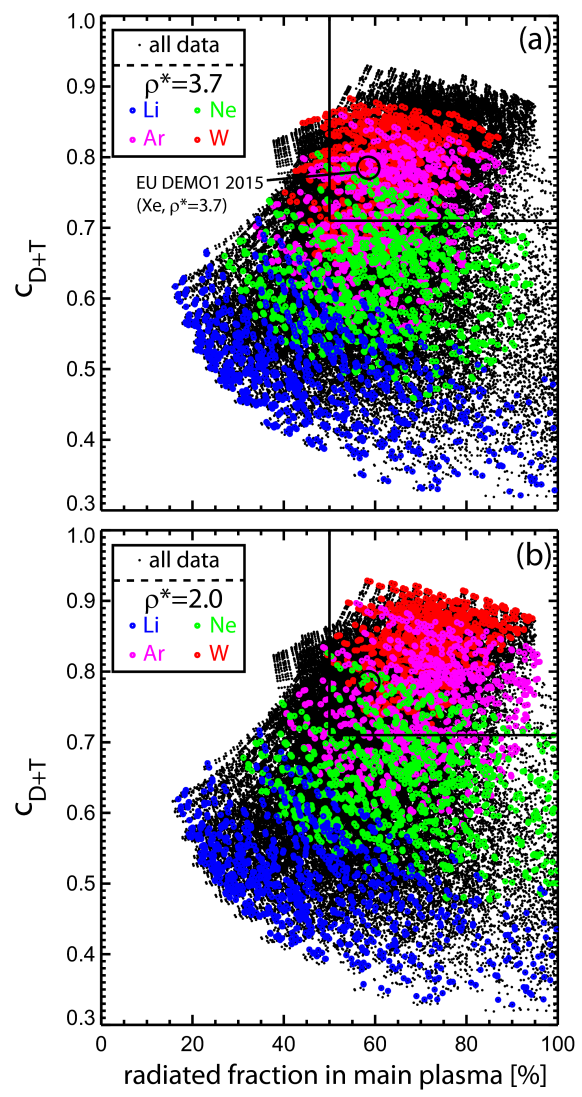

Figure 17. (colored in online-version) (a) The fuel concentration $c_{D+T}$ versus the radiated fraction for all data points of the database (black), while the data for a few impurities at $\rho^{*}=3.7$ are highlighted (colored symbols). (b) Same as (a), but the highlighted data is for $\rho^{*}=2$.

figure parts for reference. Specific subsets of simulations have been highlighted with colored circles. In detail, in figure 17(a) the simulations with $\rho^{*}=3.7$ have been highlighted for the independent impurities Li (blue), Ne (green), Ar (magenta) and W (red). Most of the model calculations for Ar and $\mathrm{W}$ are within the window $f_{\text {rad }}>50 \%$ and $c_{D+T}>0.71$, where also the EU DEMO1 2015 design using Xe for radiative cooling at $\rho^{*}=3.7$ resides (larger black circle). For Ne only a few simulations with very optimistic parameters exist in the interesting operational window, while for Li no simulation achieves the proposed figure of merit. This picture does not change when assuming a better helium exhaust of $\rho^{*}=2$ (cf. figure 17(b)). For that, all impurities perform slightly better, but still the Li data points are not in the desired window of operation and only a minority of the Ne data is found there.

One might argue that an absolute value of $c_{D+T}>0.71$ is the wrong metric to judge the performance of the various impurities, as in no case $c_{D+T}=1$ is achieved. Thus, an 
alternative approach would be a comparison to the reference impurity mixture. We chose to use the EU DEMO1 2015 mixture, i.e. Xe and $\rho^{*}=3.7$ as a reference. So, for each combination of all other parameters, $c_{D+T}$ is evaluated considering the respective impurity and $\rho^{*}$ and then it is compared to $c_{D+T}$ of the corresponding plasma with Xe and $\rho^{*}=3.7$. In order to obtain the ratio of the fusion yield, the ratio of the fuel concentrations is squared and $Q / Q_{X e, \rho^{*}=3.7}$ is obtained (cf. figure 18). Again the black data points indicate all data

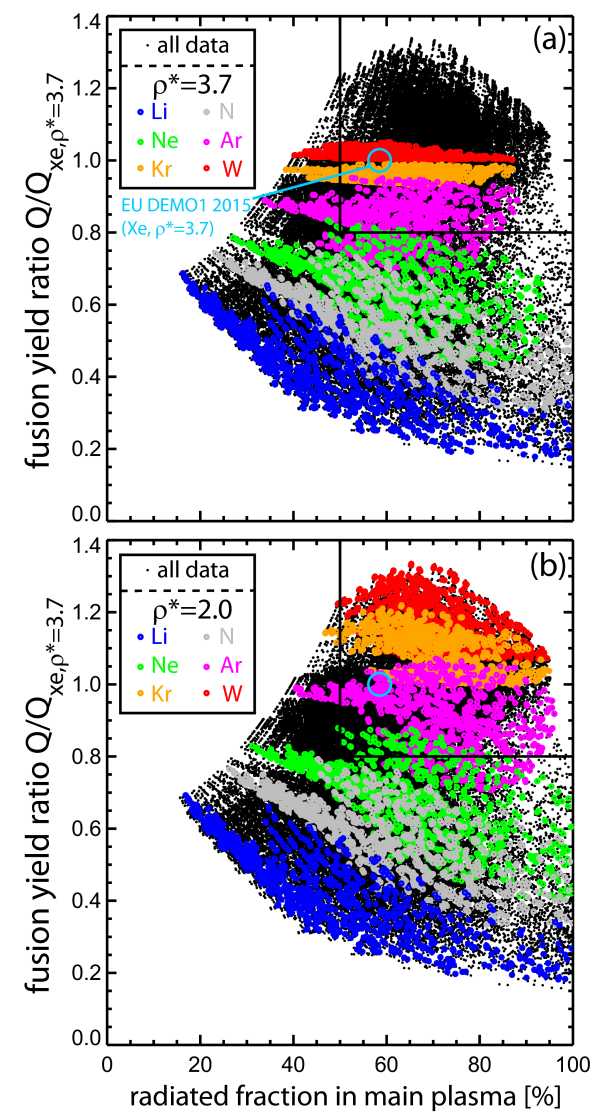

Figure 18. (colored in online-version) (a) The fusion yield $Q$ normalized by the fusion yield $Q_{X e, \rho^{*}=3.7}$, evaluated for a plasma employing Xe as only impurity at $\rho^{*}=3.7$, is depicted versus the radiated fraction for all data points of the database (black), while the data for a few impurities at $\rho^{*}=3.7$ are highlighted (colored symbols). (b) Same as (a), but the highlighted data is for $\rho^{*}=2$.

and the colored circles highlight certain impurities, i.e. Li (blue), $\mathrm{N}$ (gray), $\mathrm{Ne}$ (green), $\mathrm{Ar}$ (magenta), $\mathrm{Kr}$ (orange), and $\mathrm{W}$ (red), only. In figure 18(a) the colored data corresponds to $\rho^{*}=3.7$. A slight difference in dilution is visible between $\mathrm{W}$ and $\mathrm{Kr}$ with respect to $\mathrm{Xe}$ as the same amount of radiation corresponds to slightly less dilution for $\mathrm{W}$ and slightly more for Kr. For a reactor this means that even if Xe is used as a radiator, it can easily tolerate some $\mathrm{W}$ without any negative impact on performance. The operational window is indicated at $Q / Q_{X e, \rho^{*}=3.7}>0.8$ and $f_{\text {rad }}>50 \%$. The former boundary is again arbitrary and correponds to a $20 \%$ performance loss of the thermal power due to a different impurity 
mix as compared to the reference. The colors are relatively well ordered, which allows for a simultaneous presentation of six independent impurities. In figure 18(b), all colored data corresponds to $\rho^{*}=2.0$, allowing for lower dilution. However, this potential is only used by mid- to high-Z impurities, which clearly move up in $Q / Q_{X e, \rho^{*}=3.7}$, and the low-Z impurities with $Z \leq 10$ do not change $Q / Q_{X e, \rho^{*}=3.7}$ drastically. The underlying reason for this is, that less He allows for more content of the independent impurity. This additional content causes radiation for mid- to high- $Z$ impurities and merely weak dilution, while for impurities with $Z \leq 10$ the additional impurity content causes mostly dilution, which resembles the effect of the missing helium. In short, only strong radiators may make use of more headroom due to an improved helium exhaust in terms of fusion power performance.

At this stage further conclusion are difficult, due to the manifold features that exist in the huge dataset obtained by the multidimensional parameter scan. Nevertheless, the results of the parameter scan is made available via scaling formulae described in the following, as it provides a fast possibility to predict the impurity content of any reactor design, including the He content. Additionally, also predictions of the fusion yield and the radiated fractions can be made, which seem to be reasonably close to predictions of state-of-the-art codes. Further along these lines the formulae can be transformed into formulae for design parameters and by using scaling formulae for $\tau_{E}$ it is possible to obtain relations between fusion power and $\rho^{*}$. At least the impurity content alone provides a relative accurate value for $Z_{\text {eff }}$, which has consequences for many other aspects important to a fusion reactor independently of burn physics.

To that end scaling formulae are described in Appendix B, which are not developed to obtain insights into the underlying physics, but rather to reproduce the results of the database to a high accuracy.

Following this approach, a rather large set of coefficients has been fit to give the values, which are denoted in figure 19 with 'scaling'. As can be seen in figure 19(a), the values obtained from the scaling formulae $c_{Z \text {,scaling }}$ describe the impurity concentration quite well, while a few outliers still exists providing a factor of 1.5 too large or too small impurity content. Still, considering there are more than $10^{5}$ data points, most of the data is described quite well with deviations from the full model smaller than a factor of 1.2. The description of the He concentration $c_{H e, Z, \text { scaling }}$ by the corresponding scaling formula (cf. figure 19(b)) is of approximately similar quality. Applying the scaling formula for $c_{Z, \text { scaling }}$ and $c_{\mathrm{He}, Z, \text { scaling }}$ provides the fuel concentration

$$
c_{D+T, Z, \text { scaling }}=1-Z \cdot c_{Z, \text { scaling }}-2 \cdot c_{H e, Z, \text { scaling }},
$$

with $Z$ being the nuclear charge and not the actual ion charge. This approximation introduces only very minor deviations, as low- $Z$ impurities the ion charge is the nuclear charge and dilution from high-Z impurities is small in any case, while the ion charge is almost as large as the nuclear charge (e.g. for $\mathrm{W} q \approx 60$ at $T=10 \mathrm{keV}$ and $Z=74$ ). The dilution implies an effect on fusion power and thus, fusion yield $Q$, which can be related to the fusion yield for $\mathrm{Xe}$ as a radiatior and a $\mathrm{He}$ content corresponding to $\rho^{*}=3.7$. The resulting value of $Q / Q_{X e, \rho *=3.7}$ from the scaling is compared to that from the $0.5 \mathrm{D}$ model in figure 19(c). The comparison demonstrates that the resulting effect on fusion power is reproduced by the scaling much better than a factor of 1.2 for $Q / Q_{X e, \rho *=3.7}>0.6$, while outliers increase for even more dilution. However, such severely diluted plasmas seem anyhow irrelevant for building a fusion reactor. For $Q / Q_{X e, \rho *=3.7}<0.6$, the worst outliers are as large as a factor of 2 . Another parameter of interest is the radiated fraction $f_{\text {rad,Z,scaling }}$ from the scaling. Most of the data for $f_{\text {rad }}$ is described within a factor of 1.2 by $f_{\text {rad,Z,scaling, however, outliers are as }}$ large as approximately a factor of 1.5 . 

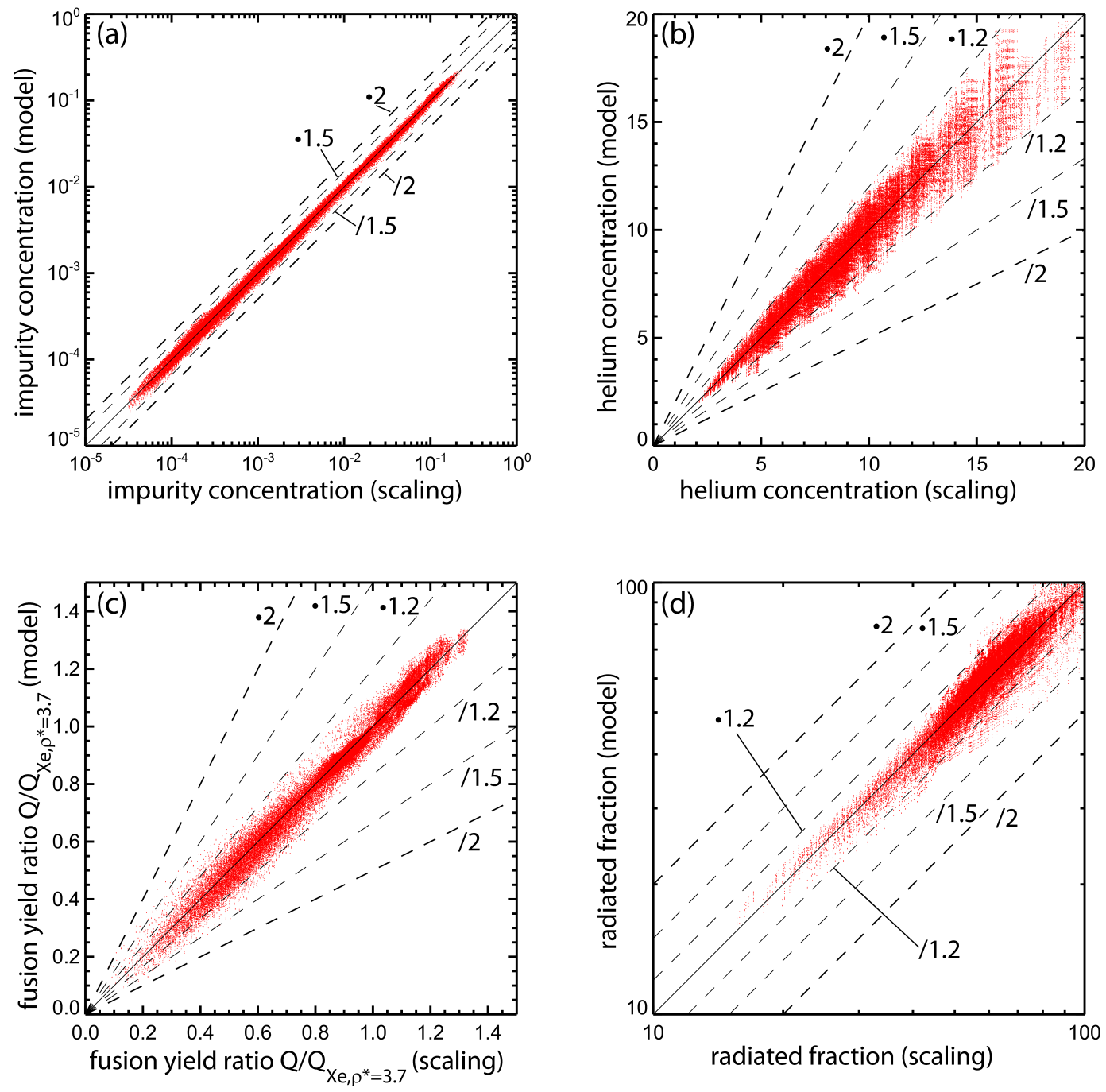

Figure 19. (colored in online-version) (a) The impurity concentration as obtained from the full 0.5D model is plotted versus the result of the scaling fromula. (b) Same as (a), but for $\mathrm{He}$ concentrations. (c) Same as (a) but for $Q / Q_{X e, \rho^{*}=3.7}$, i.e. relative factor between the fusion yield of the data and the reference fusion yield for Xe and $\rho^{*}=3.7$ at otherwise the same parameters. The dilution is calculated from the scaling presented in parts (a) and (d) Same as (a) but for radiated fraction.

It should be noted that both the $0.5 \mathrm{D}$ model and the scaling data may also be used to investigate impurity mixtures in the main plasma. The scaling formula delivers the impurity concentration $c_{k, \text { scaling }}$ for an impurity $k$ at which for the fusion plasma heating and loss meachanisms are in equilibrium. To that end the result of the scaling formula $c_{k, \text { scaling }}$ can be considered as the total budget for that impurity $k$ and this impurity may only use a fraction of its budget. The remaining head room may be filled by other impurities. This may be written 
as a linear scheme:

$$
1=\sum_{k} c_{k} / c_{k, \text { scaling }}
$$

where $c_{k}$ is the impurity concentration of the impurity $k$ and all $c_{k, s c a l i n g}$ are evaluated for the same parameters corresponding to the same fusion plasma. Note, that this linear combination is an approximation only, because the He content and thus $Q$ are not perfectly scaling linearly with the impurity mixture. However, for 429 test cases (i.e. 13 cases for all 33 impurities varying $R_{T}, R_{n}, n_{T}, n_{n}, \rho^{*}, T_{0}, n T \tau_{E}$ ) the proposed linear scheme was tested. For these test cases, $c_{N} / c_{k, \text { scaling }}=0.5$ was used and then a model handling two impurities simultaneously is compared to the linear combination proposed above. The $0.5 \mathrm{D}$ model for each individual impurity reproduced the detailed $0.5 \mathrm{D}$ model using both impurities simultaneously within $5 \%$ relative deviation as can be judged from figure 20(a). $\Delta_{r e l}$ symbolizes the relative discrepancy of the denoted quantities. For $\Delta_{r e l}$ of the impurity concentration, the half of the impurity budget of the $0.5 \mathrm{D}$ model runs (one impurity) was compared to the calculated amount of that impurity for a $0.5 \mathrm{D}$ model run handling an impurity mixture, i.e. half of the nitrogen budget and any other impurity. Then the relative difference, i.e. the difference normalized by half of the impurity budget, is evaluated and depicted in figure 20(a). Note, that in order to investigate how well linearity holds for impurity combinations, not the scaling formulae have been used, because the uncertainties of the scalings are known already.

For the same $0.5 \mathrm{D}$ model runs with an impurity mixture also the helium concentration $c_{H e, m i x}$, the fusion yield ratio $Q_{m i x} / Q_{X e, \rho *=3.7}$ and the radiated fraction $f_{\text {rad,mix }}$ may be evaluated as a linear combination of the individual results $\left(c_{H e, k, s c a l i n g}\right.$, $Q_{k, \text { scaling }} / Q_{X e, \rho *=3.7, \text { scaling }}$ and $f_{\text {rad }, k, \text { scaling }}$ ) of the impurity $k$.

$$
\begin{aligned}
c_{H e, \text { mix }} & =\sum_{k} c_{H e, k, \text { scaling }} \cdot \frac{c_{k}}{c_{k, \text { scaling }}} \\
Q_{\text {mix }} / Q_{X e, \rho *=3.7} & =\sum_{k} Q_{k, \text { scaling }} \cdot \frac{c_{k}}{c_{k, \text { scaling }}} / Q_{X e, \rho *=3.7} \\
f_{\text {rad }, \text { mix }} & =\sum_{k} f_{\text {rad }, k, \text { scaling }} \cdot \frac{c_{k}}{c_{k, \text { scaling }}}
\end{aligned}
$$

In figure 20(b-d), the deviation from linearity of the three quantities $\left(\left(c_{D+T}\right)^{2}\right.$ was chosen instead of the $Q$-ratio as the fuel concentration is responsible for the $Q$-change.) is investigated in an analogous way. The depicted $\Delta_{r e l}$ values are evaluated analogously to the described procedure for the impurity concentrations. For the 429 test cases these $\Delta_{\text {rel }}$ values are in most cases clearly below a $5 \%$ level which is much more accurate than other uncertainties. Additionally, a considerable fraction of the deviations originates from nummerical inaccuracies, as can be seen for nitrogen, which should produce a perfect match, i.e. no relative deviation in all quantities. No attempt was undertaken to minimize these uncertainties further, as the deviations are small enough and a reduction of the nummerical uncertainties would imply longer runtimes.

Thus the linear combinations are suitable way to obtain usefull numbers for the tolerable impurity concentrations in a situation where impurity mitures arise. For example, if low$\mathrm{Z}$ impurities used as divertor radiator leak from the divertor into the main plasma, while a high- $Z$ radiator is radiating in the main plasma. For such or similar considerations the impact on $c_{H e, m i x}, Q_{m i x} / Q_{X e, \rho *=3.7}$ and $f_{r a d, m i x}$ are evaluated in a relative good approximation considering the simplicity of the approach. 

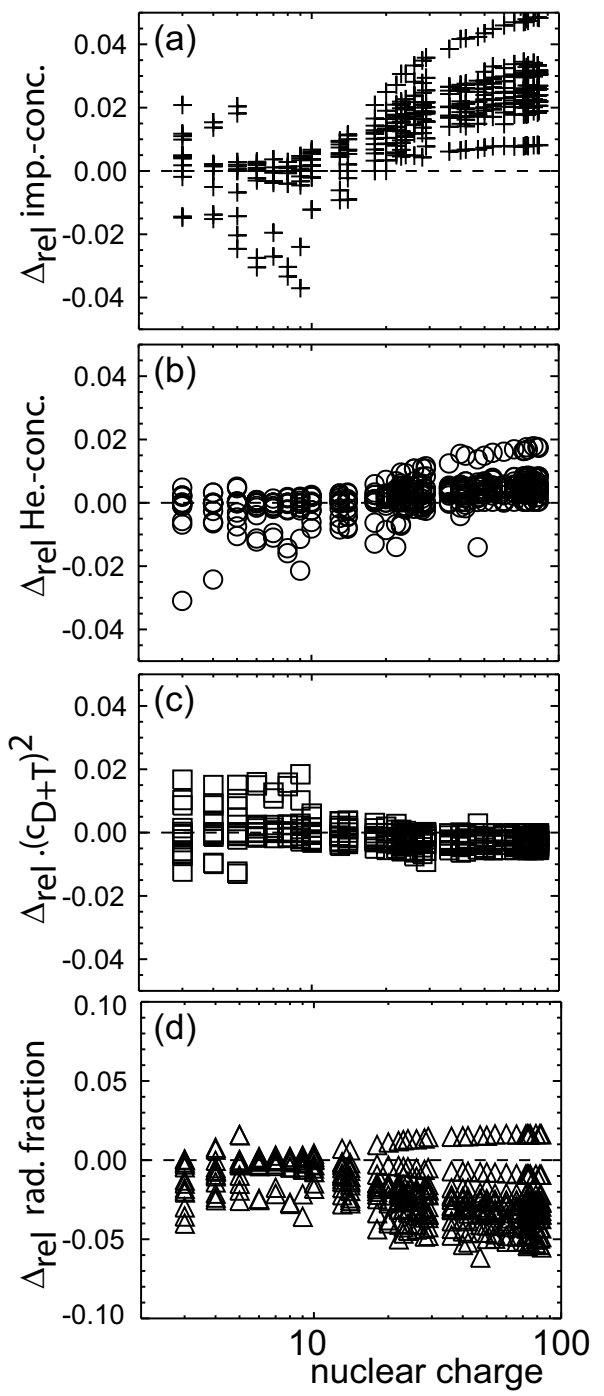

Figure 20. (a) The relative deviation of the impurity concentration tolerable along with a nitrogen content at half of the maximum nitrogen level. (b) Same as (a), giving the relative deviation of the predicted helium conentration. (c) Same as (b) but the square of the fuel concentration, which is proportional to $Q$. Same as (b) but for the radiated fraction.

\section{Conclusions \& Summary}

Before summarizing all aspects of the work, four main conclusions are drawn, which are:

- A simple and coarse reactor model is able to consider beneficial (radiative cooling fraction) and detrimental (dilution) effects of all impurities including the helium ash quite realisticly as comparisons to a full ASTRA based transport model confirm.

- The simple model allows for mapping out regions in the $n T \tau$ vs. $T$ plane in which reactor designs fullfill exhaust and economical boundary conditions. 
- The equilibrium impurity concentration for independent impurities, i.e. not the helium ash, leading to a balance of heating power and power losses scales in a wide parameter range roughly as $Z^{-2}$, where $Z$ is the nuclear charge of the impurity.

- However, the model suggests that a contamination of the main plasma by low-Z impurities $(Z<18)$ should be avoided for efficiency reasons, as the fusion gain inevitably suffers due to dilution - even more so, if the reduced plasma heating caused by dilution leads to a reduction of the plasma core temperature. The above conclusion is also true, if an alternative assumption of constant plasma pressure, i.e. increased core temperature for diluted plasmas, is employed. The temperature rise is in all cases clearly too small for compensating the fusion yield reduction due to dilution.

In more detail, the content of the present work can be summarized as follows. The first part is concerned with the calculation of atomic data for 35 different elements including most of the elements that may be found in today's and future fusion experiments. The calculated atomic data provide ionization and recombination rate coefficients and thus, the ionization equilibrium. Additionally, Bremsstrahlung, radiative recombination, radiation due to dielectronic recombination and line radiation are considered. The present work employs the same models as were used for $\mathrm{W}$ in [4] for all considered elements. This leads to atomic data, which is of reasonable quality for high-Z elements and baseline quality for low-Z impurities. Most importantly the data set is consistent and allows to study the limits of impurities in reactor plasmas, while code or model specific effects are excluded. As the data fills many holes for mid-Z to high-Z elements also spectral predictions will be made available.

The second part of the present work investigates the limit of impurities for a variety of reactor models. A 0D power balance between fusion $\alpha$-heating and losses from radiation and transport is investigated for 33 independent (i.e. not helium or hydrogen) impurities as a function of the ratio of helium confinement time including recycling $\tau_{H e}^{*}$ over energy confinement time $\tau_{E}$, i.e. $\rho^{*}$. The maximum impurity concentrations are determined by scanning the whole $n T \tau_{E}$ versus $T$ plane. It is found that if low-Z elements dominate, the maximum impurity level is achieved at relatively low $T<30 \mathrm{keV}$, while for dominating high-Z impurities, the maximum impurity level occurs at $T>40 \mathrm{keV}$. In cases where helium dominates the balance $\left(\rho^{*}>10\right)$ the maximum impurity level of the independent impurity is obtained at $T \approx 15 \mathrm{keV}$. The results of the $0 \mathrm{D}$ model could be summarized in a relatively simple power law. The maximum impurity level exhibits an approximate scaling with $1 / Z^{2}$, where $Z$ is the nuclear charge of the impurity.

As a next step profile effects have been included in the power balance, and thus, the resulting model is called $0.5 \mathrm{D}$ model. To that end self similar temperature and density profiles with a peaking and a shape parameter have been implemented such that the basic mechanisms of the $0 \mathrm{D}$ model could still be used as a very good approximation. The comparisons to the OD model show that at moderate profile peaking and reactor relevant helium confinement the effect of the profile consideration on the maximum impurity is moderate, i.e. smaller than about a factor of 1.5.

The $0.5 \mathrm{D}$ reactor model is then further extended taking auxiliary heating and additional loss mechanisms such as synchrotron radiation into account via a finite fusion yield $Q$, which is labelled $Q_{n e t}$ in case additional losses are considered. In the course of approaching realistic reactor properties, radiated fractions and dilution of the fuel are investigated and it is found, that a specific subarea of the full $n T \tau_{E}$ versus $T$ plane is best suited to provide a power exhaust solution and a dilution such that a reactor seems economically feasibile. This area symbolizing a possible operation window is largest for high-Z impurities at low as possible $\rho^{*}$ values providing large radiative fractions at small dilution. The operational point of the 
EU DEMO1 2015 design using high-Z impurities resides in that window.

As a further step the influence of the impurity mix on the reactor parameters and performance are studied. To that end the EU DEMO1 2015 design is used as a reference design, where the design includes the original impurity mix and thus $c_{D+T, \text { ref }}$ (deuterium+tritium concentration) and $c_{t o t, r e f}$ (the particle concentration including electrons, ions and impurity ions) as well as the $T$ and $n$ profiles. This allows for evaluation of $Q$ for a fixed $P_{\text {aux }}$ within the $0.5 \mathrm{D}$ model when the dilution is changed via a change in the impurity mix. This results in quite obvious changes of $Q$ up to about a factor of two when low-Z impurities are dominant in the reactor plasma. Only a small fraction of this decrease in $Q$ is compensated if the assumption of constant plasma pressure is employed leading to an increase of $T$ for diluted plasmas. Even worse are the predictions for low-Z impurities of a realistic 1D ASTRA transport simulation in which dilution of the fusion fuel leads to a decrease of the heat fluxes and thus, plasma temperature. When comparing the 1D ASTRA model to the $0.5 \mathrm{D}$ model, this qualitative difference shows up mostly in a decreased tolerance of low$\mathrm{Z}$ impurities in the 1D ASTRA run as compared to the $0.5 \mathrm{D}$ model. For high-Z impurities and reactor relevant $1<\rho^{*}<10$ the predicted impurity concentrations of the 1D ASTRA simulation and the $0.5 \mathrm{D}$ model agree within $10 \%$ and also the predicted $Q$ values and helium concentrations are within an agreement of $\approx 10 \%$.

As the most detailed version of the $0.5 \mathrm{D}$ model reproduced the impurity concentrations, the helium content and the fusion yield of the 1D ASTRA model reasonably well, a parameter scan was performed spanning the most interesting region for $\langle n\rangle T_{0} \tau_{E}, T_{0}, Q, \rho^{*}, T_{0} /\langle T\rangle$, $n_{0} /\langle n\rangle$ and the coefficients $n_{T}$ and $n_{n}$, which define the profile shapes of density and temperature. As a result a database with more than $10^{5}$ datapoints was set up, which was used to develop scaling formulae, which reproduce the impurity and helium concentration, the fusion yield and the radiated fraction. The database was analyzed in terms of fusion yield and radiated fraction and clearly the low-Z impurity content prevents access to the economical interesting region (i.e. high fusion yield and good power exhaust). For high-Z elements the radiating impurity is of minor importance such that the performance of plasmas using $\mathrm{Kr}, \mathrm{Xe}$ or $\mathrm{W}$ as radiators are almost equally good. As a consequence, a part of the impurity budget may be used up by $\mathrm{W}$ without any negative impact on performance. The database results may also be used to consider impurity mixtures. To that end a linear combination of impurity contents allows for the prediction of all performance parameters.

It should be noted that all predictions presented in this work are based on the assumption that impurity concentration profiles are flat within the confined region of the fusion plasma. This assumption might be only slightly violated by independent impurities, however, in case of helium, it is clear that the location of the source will lead to a tendency of peaked helium profiles if transport does not strongly counteract. Generally, hollow impurity profiles are allways beneficial, i.e. increasing $Q$ of a power plant, while meeting power exhaust conditions. This even extends beyond the separatrix, as power exhaust in the plasma edge requires impurities in the divertor plasma, which in the best case are retained there. Anyhow, given that the variation within the impurity concentration profiles is relatively small, the present work suggests, that the sustainable impurity density in a future reactor may be estimated to a relative good accuracy by only knowing basic parameters of the main plasma.

\section{Acknowledgemt}

This work has been carried out within the framework of the EUROfusion Consortium and has received funding from the Euratom research and training programme 2014 - 2018 under grant 
agreement No 633053. The views and opinions expressed herein do not necessarily reflect those of the European Commission.

\section{References}

[1] D. Post et al., At. Data Nucl. Data Tables 20, 397 (1977).

[2] H. P. Summers et al., Plasma Physics and Controlled Fusion 48, 263 (2006).

[3] T. Nakano, Journal of Nuclear Materials 415, S327 (2011), proceedings of the 19th International Conference on Plasma-Surface Interactions in Controlled Fusion.

[4] T. Pütterich et al., Plasma Physics and Controlled Fusion 50, 085016 (2008).

[5] T. Pütterich et al., Nuclear Fusion 50, 025012 (9pp) (2010).

[6] A. Foster, Technical report, University of Strathclyde, Glasgow, UK, doctoral thesis at Department of Physics, University of Strathclyde, Glasgow, UK (2008).

[7] A. Sasaki and I. Murakami, Journal of Physics B: Atomic, Molecular and Optical Physics 46, 175701 (2013).

[8] I. Murakami et al., Nuclear Fusion 55, 093016 (2015).

[9] H. Lux, R. Kemp, D. Ward, and M. Sertoli, Fusion Engineering and Design 101, 42 (2015).

[10] R. Wenninger et al., Nuclear Fusion 57, 016011 (2017).

[11] W. J. Karzas and R. Latter, Astrophysical Journal Supplements VI, 167 (1961).

[12] R. S. Sutherland, Monthly Notices of the Royal Astronomical Society 300, 321 (1998).

[13] H. P. Summers, The ADAS User Manual, version 2.6 http://adas.phys.strath.ac.uk (2004).

[14] S. D. Loch et al., Physical Review A (Atomic, Molecular, and Optical Physics) 72, 052716 (2005).

[15] R. D. Cowan, The Theory of Atomic Structure and Spectra (Los Alamos Series in Basic and Applied Sciences) (University of California Press, California, 1981).

[16] S. S. Henderson et al., Plasma Physics and Controlled Fusion 59, 055010 (2017).

[17] A. Burgess, Astrophysical Journal 141, 1588 (1965).

[18] N. R. Badnell et al., Astronomy and Astrophysics 406, 1151 (2003).

[19] F. Albajar, J. Johner, and G. Granata, Nuclear Fusion 41, 665 (2001).

[20] M. Siccinio et al., Nuclear Fusion 58, 016032 (2018).

[21] D. Reiter, G. H. Wolf, and H. Kever, Nuclear Fusion 30, 2141 (1990).

[22] H.-S. Bosch and G. M. Hale, Nuclear Fusion 32, 611 (1992), erratum in 33(12), 1919 (1993).

[23] H.-S. Bosch et al., Plasma Physics and Controlled Fusion 39, 1771 (1997).

[24] G. N. Pereverzev, C. Angioni, A. G. Peeters, and O. V. Zolotukhin, Nuclear Fusion 45, 221 (2005).

[25] M. Kovari et al., Fusion Engineering and Design 89, 3054 (2014).

[26] M. Kovari et al., Fusion Engineering and Design 104, 9 (2016).

[27] G. V. Pereverzev et al., Technical Report No. 5/42, IPP, Garching, Germany, (1991).

[28] E. Fable et al., Plasma Physics and Controlled Fusion 55, 124028 (2013).

[29] R. Wenninger et al., Nuclear Fusion 54, 114003 (2014).

[30] E. Fable, R. Wenninger, and R. Kemp, Nuclear Fusion 57, 022015 (2017).

[31] G. M. Staebler, J. E. Kinsey, and R. E. Waltz, Physics of Plasmas 14, 055909 (2007). 


\section{Appendix A. Properties of Considered Profiles and Derivation of Correction Factors}

First it is worthwhile to note that for a given set $T_{0}, n_{T}$ and $R_{T}$ (or $n_{e, 0}, n_{n}$ and $R_{n}$ ) the edge Temperature $T_{\text {edge }}$ ( edge density $n_{e, e d g e}$ ) is determined and independent of $a$. This is demonstrated for the temperature profile $T(r / a)=T(x)=T_{0}\left(1-\frac{T_{0}-T_{\text {edge }}}{T_{0}} x^{n_{T}}\right)$. The volume average

$$
\begin{array}{rl}
\langle T\rangle=\frac{2 \pi L a^{2}}{V(1)} \int_{0}^{1} T(x) x & \mathrm{~d} x=2\left[\frac{1}{2} T_{0} x^{2}-\frac{1}{\left(n_{T}+2\right)}\left(T_{0}-T_{\text {edge }}\right) x^{n_{T}+2}\right]_{0}^{1}= \\
=T_{0} & -\frac{2}{n_{T}+2}\left(T_{0}-T_{\text {edge }}\right)
\end{array}
$$

Thus,

$$
T_{\text {edge }}=\frac{n_{T}+2}{2}\left(\langle T\rangle-T_{0}\right)+T_{0}=T_{0} \frac{n_{T}+2-n_{T} R_{T}}{2 R_{T}}
$$

independently of $a$. Furthermore,

$$
\begin{gathered}
T(x)=T_{0}\left(1-\frac{T_{0}-T_{0} \frac{n_{T}+2-n_{T} R_{T}}{2 R_{T}}}{T_{0}} x^{n_{T}}\right)=\langle T\rangle R_{T}\left[1-\left(\frac{n_{T}+2}{2} \frac{R_{T}-1}{R_{T}}\right) x^{n_{T}}\right]= \\
=\langle T\rangle G\left(R_{T}, n_{T}, x\right)
\end{gathered}
$$

defines the dimensionless function $G$, which can also be used to express the density profile

$$
n(r)=\langle n\rangle G\left(R_{n}, n_{n}, x\right) \quad .
$$

It is important to note that the volume average of any quantity $X$, which is only a funtion of $n(x)$ and $T(x)$ profiles with above mentioned properties, is trivially independent of the plasma size, since

$$
\langle X\rangle=2 \int_{0}^{1} X(T(x), n(x)) x \mathrm{~d} x
$$

In the following, it is demonstrated how the consideration of profiles can be taken into account via simple correction factors within the $0 \mathrm{D}$ equations for the power and He particle balance. For the $\alpha$-heating we obtain the correction factor $F_{\text {heating }}$ by comparing:

$$
2 E_{\alpha} \int_{0}^{1}\left(\frac{c_{D+T}(T(x)) \cdot n_{e}(x)}{2}\right)^{2}\langle\sigma v\rangle x \mathrm{~d} x=E_{\alpha}\left(\frac{c_{D+T}(\langle T\rangle) \cdot\left\langle n_{e}\right\rangle}{2}\right)^{2}\langle\sigma v\rangle_{\langle T\rangle} F_{\text {heating }}
$$

Thus,

$$
F_{\text {heating }}=2 \frac{\int_{0}^{1}\left(c_{D+T}(T(x)) \cdot G\left(R_{n}, n_{n}, x\right)\right)^{2}\langle\sigma v\rangle x \mathrm{~d} x}{c_{D+T}^{2}(\langle T\rangle)\langle\sigma v\rangle_{\langle T\rangle}}
$$

where $c_{D+T}$ is radially not constant, because $q$ changes with $T$. Note, that $F_{\text {heating }}$ is independent of $a$ and can be evaluated for known $T_{0}, R_{T}, n_{T}, R_{n}$ and $n_{n}$, while the absolute density $n_{0}$ has cancelled.

For the radiative cooling we can obtain a correction factor for each contributing element separately, i.e. $F_{r a d, H}, F_{r a d, H e}$ and $F_{r a d, Z}$ by comparing:

$$
2 \int_{0}^{1} c_{D+T}(T(x)) \cdot n_{e}^{2}(x) L_{H}(T(x)) x \mathrm{~d} x=c_{D+T}(\langle T\rangle) \cdot\left\langle n_{e}\right\rangle^{2} L_{H}(\langle T\rangle) F_{r a d, H}
$$




$$
\begin{gathered}
2 \int_{0}^{1} c_{H e} n_{e}^{2}(x) L_{H e}(T(x)) x \mathrm{~d} x=c_{H e}\left\langle n_{e}\right\rangle^{2} L_{H e}(\langle T\rangle) F_{r a d, H e} \\
2 \int_{0}^{1} c_{Z} n_{e}^{2}(x) L_{Z}(T(x)) x \mathrm{~d} x=c_{Z}\left\langle n_{e}\right\rangle^{2} L_{Z}(\langle T\rangle) F_{r a d, Z}
\end{gathered}
$$

Thus,

$$
\begin{gathered}
F_{r a d, H}=2 \frac{\int_{0}^{1} c_{D+T}(T(x)) \cdot G^{2}\left(R_{n}, n_{n}, x\right) L_{H}(T(x)) x \mathrm{~d} x}{c_{D+T}(\langle T\rangle) L_{H}(\langle T\rangle)} \\
F_{r a d, H e}=2 \frac{\int_{0}^{1} G^{2}\left(R_{n}, n_{n}, x\right) L_{H e}(T(x)) x \mathrm{~d} x}{L_{H e}(\langle T\rangle)} \\
F_{r a d, Z}=2 \frac{\int_{0}^{1} G^{2}\left(R_{n}, n_{n}, x\right) L_{Z}(T(x)) x \mathrm{~d} x}{L_{Z}(\langle T\rangle)}
\end{gathered}
$$

Finally, for transport losses we obtain the correction factor $F_{\text {transport }}$ by comparing:

$$
\frac{2}{\tau_{E}} \int_{0}^{1} \frac{3}{2} c_{t o t}(T(x)) \cdot n_{e}(x) k_{B} T(x) x \mathrm{~d} x=\frac{\frac{3}{2} c_{t o t}(\langle T\rangle) \cdot\left\langle n_{e}\right\rangle k_{B}\langle T\rangle}{\tau_{E}} F_{\text {transport }}
$$

Thus,

$$
F_{\text {transport }}=2 \frac{\int_{0}^{1} c_{t o t}(T(x)) \cdot G\left(R_{n}, n_{n}, x\right) T(x) x \mathrm{~d} x}{c_{t o t}(\langle T\rangle)\langle T\rangle}
$$

For the evaluation of the He balance the correction factor is $F_{\text {heating }}$ for the He source, while the sink does not require a correction factor:

$$
\left(\frac{c_{D+T}(\langle T\rangle) \cdot\left\langle n_{e}\right\rangle}{2}\right)^{2}\langle\sigma v\rangle_{\langle T\rangle} F_{\text {heating }}=\frac{c_{H e}\left\langle n_{e}\right\rangle}{\tau_{H e}^{*}} \quad \text { (0.5D He balance) }
$$

Unfortunately, the application of the correction factors still yields a fundamental issue. Due to the occurrence of a profile dependent impurity charge $q$, the correction factors $F_{\text {heating }}, F_{\text {rad,H }}$ and $F_{\text {transport }}$ depend non-trivially on $c_{H e}$. This dependence is contained in $c_{D+T}$ and $c_{t o t}$. Such a dependence is preventing the determination of $c_{H e}$ along the lines presented in [21], i.e. the OD model. However, this dependence may be removed by approximating $q(T(x))$ by $q(\langle T\rangle)$. The introduced error of this approximation can be estimated by repeating all following calculations and fixing $q(T(r))$ to $q\left(T_{0}\right)$ or $q\left(T_{\text {edge }}\right)$ instead of $q(\langle T\rangle)$. This has been done and all deviations in the evaluated tolerable impurity concentrations are below a relative size of $0.7 \%$ on the evaluated values and thus, are considered unimportant. Additionally, the effect of this simplification on the term $c_{D+T}=$ $\left(1-q c_{Z}-2 c_{H e}\right)$ or $c_{t o t}=\left(2-(q-1) c_{Z}-c_{H e}\right)$ may be checked with realistic values for $c_{Z}$ and $c_{H e}$. As $c_{D+T}$ is more crucial and because $c_{t o t}$ is also very similar to $c_{D+T}$ the focus is now put on $c_{D+T}$ only. The relative change of this term $\delta_{\text {rel }}$ (cf. below) for a linear, peaked temperature profile, i.e. $n_{T}=1$ with $R_{T}=2.5$ has been investigated using the $0 \mathrm{D}$-results for $c_{Z}$ and $c_{H e}$. In detail,

$$
\delta_{r e l}=\frac{c_{D+T}(\langle T\rangle)-c_{D+T}\left(T_{0}\right)}{c_{D+T}(\langle T\rangle)}=\frac{\left(q\left(T_{0}\right)-q(\langle T\rangle)\right) c_{Z}}{1-q(\langle T\rangle) c_{Z}-2 c_{H e}}
$$

The largest deviations $\delta_{\text {rel }}$ are found at small temperatures and small $\rho^{*}$. At $T_{0}=4 \mathrm{keV}$ and $\rho^{*}=0.5$ using the maximum argon (Ar) concentration found by the $0 \mathrm{D}$ model, i.e. $1.6 \%$ 
Determination of the Tolerable Impurity Concentrations in a Fusion Reactor using a Consistent Set of Cooling Factors 42

\begin{tabular}{l|cccccccc} 
Parameter & $\langle n\rangle T_{0} \tau_{E}$ & $T_{0}$ & $Q_{n e t}$ & $\rho^{*}$ & $T_{0} /\langle T\rangle$ & $n_{0} /\langle n\rangle$ & $n_{T}$ & $n_{n}$ \\
\hline dimensions & $10^{22} \mathrm{keV} \mathrm{m}^{-3} \mathrm{~s}$ & $\mathrm{keV}$ & - & - & - & - & - & - \\
\hline abrev. below & $n T \tau$ & $T$ & $Q$ & $\rho$ & $R_{T}$ & $R_{n}$ & $n_{T}$ & $n_{n}$
\end{tabular}

Table B1. Input parameters, their dimensions and abreviations as used in below formulae.

(tolerable at $36.5 \mathrm{keV}$ ), and the associated $c_{H e}=0.051$ the deviation $\delta_{r e l}=0.032$. This is the worst case for all temperatures, impurities and $\rho^{*}$. For more realistic temperatures $15 \mathrm{keV}<T_{0}<100 \mathrm{keV}$ and $\rho^{*}=5$ the $\delta_{\text {rel }}$ stays below $0.5 \%$ for all impurity elements. Thus, we consider the approximation of constant impurity charge appropriate and simplify the above correction factors to

$$
\begin{gathered}
F_{\text {heating }}=2 \frac{\int_{0}^{1}\left(c_{D+T}(\langle T\rangle) \cdot G\left(R_{n}, n_{n}, x\right)\right)^{2}\langle\sigma v\rangle x \mathrm{~d} x}{c_{D+T}^{2}(\langle T\rangle)\langle\sigma v\rangle_{\langle T\rangle}}=2 \frac{\int_{0}^{1} G^{2}\left(R_{n}, n_{n}, x\right)\langle\sigma v\rangle x \mathrm{~d} x}{\langle\sigma v\rangle_{\langle T\rangle}} \\
F_{\text {rad }, H}=2 \frac{\int_{0}^{1} c_{D+T}(\langle T\rangle) \cdot G^{2}\left(R_{n}, n_{n}, x\right) L_{H}(T(x)) x \mathrm{~d} x}{c_{D+T}(\langle T\rangle) L_{H}(\langle T\rangle)}=2 \frac{\int_{0}^{1} G^{2}\left(R_{n}, n_{n}, x\right) L_{H}(T(x)) x \mathrm{~d} x}{L_{H}(\langle T\rangle)} \\
F_{\text {transport }}=2 \frac{\int_{0}^{1} c_{t o t}(\langle T\rangle) \cdot G\left(R_{n}, n_{n}, x\right) T(x) x \mathrm{~d} x}{c_{\text {tot }}(\langle T\rangle)\langle T\rangle}=2 \frac{\int_{0}^{1} G\left(R_{n}, n_{n}, x\right) T(x) x \mathrm{~d} x}{\langle T\rangle} .
\end{gathered}
$$

\section{Appendix B. Scaling Formulae Predicting the Impurity Concentration and Radiated Fraction}

In the following it will be described how the values $c_{Z, \text { scaling }}, c_{H e, Z, \text { scaling }}$ and $f_{\text {rad,Z,scaling }}$ are obtained from a scaling formula with the input valiables listed in table B1. These input parameters are the users choice and need to be put into the three scaling formulae below.

$$
\begin{aligned}
& c_{Z, \text { scaling }}= \\
& 10^{A_{0}} \cdot(n T \tau)^{A_{1}} \cdot\left(\frac{T}{30}\right)^{A_{2}+A_{3} \rho} \cdot\left(1-\frac{5}{Q}\right)^{A_{4}} \cdot \rho^{A_{5}+A_{6} \frac{T}{30}} \cdot\left(\frac{R_{T}}{R_{n}}\right)^{A_{7}+A_{8} \frac{T}{30}} \cdot n_{T}^{A_{9}} \cdot n_{n}^{A_{10}} \\
& c_{H e, Z, \text { scaling }}= \\
& B_{0} \cdot(n T \tau)^{B_{1}} \cdot T^{B_{2}} \cdot \rho^{B_{3}+B_{4}(n T \tau)+B_{5} \frac{T}{30}} \cdot R_{T}^{B_{6}} \cdot R_{n}^{B_{7}+B_{8}(n T \tau)+B_{9} \frac{T}{30}+B_{10} \rho} \cdot n_{n}^{B_{11}} \\
& f_{\text {rad }, Z, \text { scaling }}= \\
& C_{0} \cdot(n T \tau)^{C_{1}+C_{2} \frac{T}{30}} \cdot\left(\frac{T}{30}\right)^{C_{3}+C_{4}(n T \tau)} \cdot\left(1-\frac{5}{Q}\right)^{C_{5}} \cdot \rho^{C_{6}} \cdot R_{T}^{C_{7}} \cdot R_{n}^{C_{8}+C_{9}(n T \tau)+C_{10}\left(\frac{T}{30}\right)}
\end{aligned}
$$

For a prediction of $c_{Z, \text { scaling }}, c_{H e, Z, \text { scaling }}$ and $f_{\text {rad,Z, scaling }}$ the coefficients $A_{0}$ to $A_{10}, B_{0}$ to $B_{11}$ and $C_{0}$ to $C_{10}$ are needed, which are dependent on the nuclear charge of the impurity $Z$. In order to increase the accuracy of the description, each coefficient is described as a polynomial in $Z$ of order 6 or 8 . The coefficients can be obtained via following multiplication:
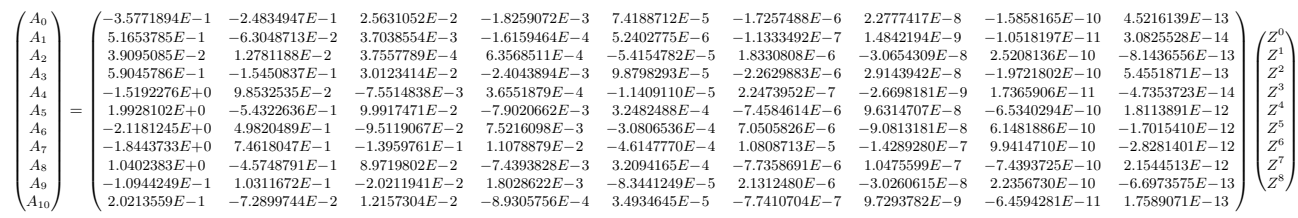
Determination of the Tolerable Impurity Concentrations in a Fusion Reactor using a Consistent Set of Cooling Factors 43

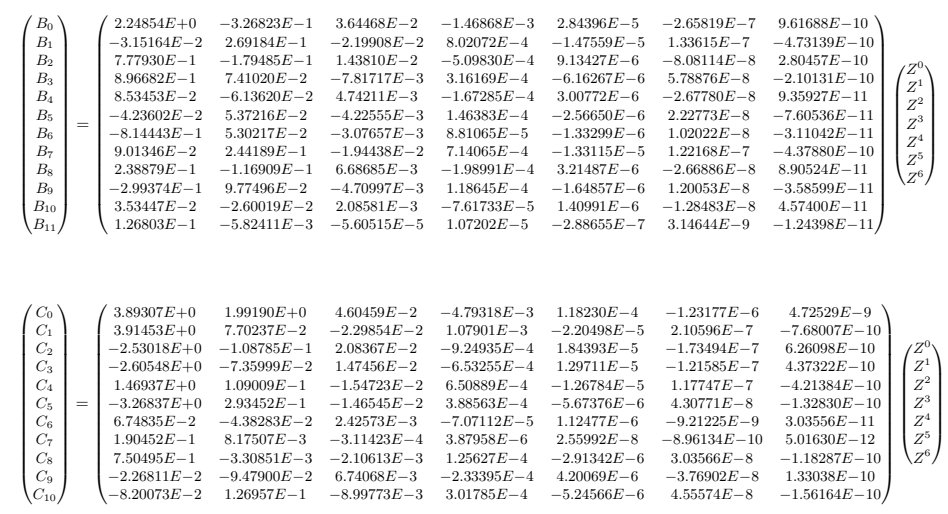

